\title{
Modular Syntheses of Phenanthroindolizidine Natural
}

\section{Products}

\author{
Young-In $\mathrm{Jo}^{1}$, Martin D. Burke ${ }^{2, *}$, and Cheol-Hong Cheon ${ }^{1, *}$ \\ ${ }^{1}$ Department of Chemistry, Korea University, 145 Anam-ro, Seongbuk-gu, Seoul 02841, Republic of Korea \\ ${ }^{2}$ Department of Chemistry and Carle Illinois College of Medicine, University of Illinois at Urbana-
} Champaign, 600 S. Mathews Ave., Urbana, IL 61801, U. S. A.

\section{Table of Contents}

1. General Information

2. Synthesis of Building Blocks S3

3. Total Synthesis of Phenanthroindolizidine Alkaloids S6

4. References S20

5. Spectroscopic Data $\mathrm{S} 21$ 


\section{General Information}

All reactions were carried out in oven-dried glassware in an open flask, unless otherwise noted. Except as otherwise indicated, all reactions were magnetically stirred and monitored by analytical thin layer chromatography (TLC) using pre-coated silica gel glass plates $(0.25 \mathrm{~mm})$ with F254 indicator. Visualization was accomplished by UV light $(254 \mathrm{~nm})$, with a combination of potassium permanganate and/or phosphomolybdic acid solution as an indicator. Flash column chromatography was performed using silica gel $60(230-400$ mesh). Yields refer to chromatographically and spectroscopically pure compounds, unless otherwise noted. Commercial grade reagents and solvents were used without further purification. Boronic acids $\mathbf{8}$ and dibromopyridines 11 were purchased from commercial suppliers and used without further purification unless otherwise noted. orthoBrominated MIDA (N-methyliminodiacetic acid) boronate 9 was prepared via the procedure reported in the literature. ${ }^{1}{ }^{1} \mathrm{H}$ NMR and ${ }^{13} \mathrm{C}$ NMR spectra were recorded at $500 \mathrm{MHz}$ and $125 \mathrm{MHz}$ spectrometers, respectively. Tetramethylsilane $(\delta: 0.0 \mathrm{ppm})$ and residual NMR solvents, either $\mathrm{CDCl}_{3}\left(\delta_{\mathrm{H}}: 7.26 \mathrm{ppm}, \delta_{\mathrm{C}}: 77.16 \mathrm{ppm}\right)$ or DMSO-d ${ }_{6}\left(\delta_{\mathrm{H}}: 2.50 \mathrm{ppm}, \delta_{\mathrm{C}}: 39.52 \mathrm{ppm}\right)$, were used as internal standards for ${ }^{1} \mathrm{H}$ NMR and ${ }^{13} \mathrm{C}$ NMR spectra, respectively. The proton spectra are reported as follows: $\delta$ (position of proton, multiplicity, coupling constant $J$, number of protons). Multiplicities are indicated by s (singlet), d (doublet), t (triplet), q (quartet), p (quintet), h (septet), m (multiplet) and br (broad). High resolution mass spectra (HRMS) were recorded on quadrupole time-of-flight mass spectrometer (QTOF-MS) using electrospray ionization (ESI) as an ionization method. Infrared spectra were measured by using attenuated total reflectance (ATR) mounted Bruker Alpha FT-IR spectrometer. 


\section{Synthesis of Building Blocks}

\section{2-1. Synthesis of ortho-Bromoaryl MIDA Boronate 9}<smiles>COc1ccc(Br)c(OC)c1</smiles>

$8 a$<smiles>CN(CC(=O)O)CC(=O)O</smiles>
$4 \AA \mathrm{MS}, \mathrm{DMF}, 120^{\circ} \mathrm{C}$

2. N-bromosuccinimide DMF, rt

To a solution of $\mathbf{8 a}(3.6 \mathrm{~g}, 20 \mathrm{mmol})$ in $200 \mathrm{~mL}$ of DMF were added $\mathrm{N}$-methyliminodiacetate $(4.4 \mathrm{~g}$, $30 \mathrm{mmol}$ ) and molecular sieves (4 ̊́, $10 \mathrm{~g}$ ). The reaction mixture was heated to $120^{\circ} \mathrm{C}$ under nitrogen atmosphere and monitored by TLC. After complete consumption of boronic acid 8a, the reaction mixture was cooled to room temperature and molecular sieves were filtered off. Then the reaction mixture was concentrated to provide a crude mixture of MIDA boronate of boronic acid $\mathbf{8 a}$, which was used for the next step without further purification. To a solution of a crude mixture of MIDA boronate of 8a in DMF (200 mL) was added a solution of N-bromosuccinimide (NBS, $5.3 \mathrm{~g}$, $30 \mathrm{mmol})$ in DMF $(20 \mathrm{~mL})$. The reaction mixture was stirred at room temperature until all MIDA boronate of $\mathbf{8 a}$ was consumed. After the complete consumption of MIDA boronate of $\mathbf{8 a}$, the reaction mixture was quenched with $100 \mathrm{~mL}$ of saturated $\mathrm{Na}_{2} \mathrm{~S}_{2} \mathrm{O}_{3}$ aqueous solution. Upon addition of additional $200 \mathrm{~mL}$ of $\mathrm{H}_{2} \mathrm{O}$ to the reaction mixture, the white precipitate was formed, collected by filtration and washed with $\mathrm{H}_{2} \mathrm{O}$. The resulting white solid was identified as compound 9 (7.0 g, 94\%). Spectroscopic data was in good agreement with the literature. ${ }^{1}$

${ }^{1}$ H NMR (500 MHz, DMSO-d 6 , ppm): $\delta 7.12(\mathrm{~s}, 1 \mathrm{H}), 7.04(\mathrm{~s}, 1 \mathrm{H}), 4.39(\mathrm{~d}, J=17.39 \mathrm{~Hz}, 2 \mathrm{H}), 4.13$ $(\mathrm{d}, J=17.40 \mathrm{~Hz}, 2 \mathrm{H}), 3.78(\mathrm{~s}, 3 \mathrm{H}), 3.76(\mathrm{~s}, 3 \mathrm{H}), 2.67(\mathrm{~s}, 3 \mathrm{H})$.

\section{2-2. Synthesis of 3-(4-Bromopyridin-2-yl)prop-2-yn-1-ol (10a)}

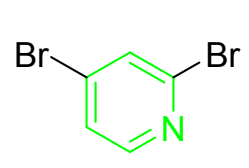

$11 \mathrm{a}$

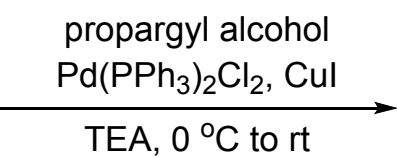

TEA, $0{ }^{\circ} \mathrm{C}$ to $\mathrm{rt}$

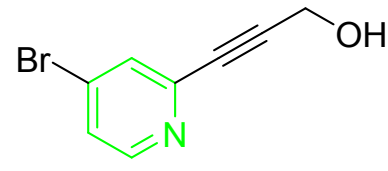

$10 \mathrm{a}$ 
A solution of 11a $(2.4 \mathrm{~g}, 10 \mathrm{mmol})$, propargyl alcohol $(0.58 \mathrm{~mL}, 10 \mathrm{mmol}), \mathrm{Pd}\left(\mathrm{PPh}_{3}\right)_{2} \mathrm{Cl}_{2}(0.21 \mathrm{~g}$, $0.30 \mathrm{mmol})$ and copper(I) iodide $(57 \mathrm{mg}, 0.30 \mathrm{mmol})$ in $20 \mathrm{~mL}$ of triethylamine was stirred at $0{ }^{\circ} \mathrm{C}$ under nitrogen atmosphere. The reaction mixture was warmed to room temperature and allowed to stir at the same temperature. After $2 \mathrm{~h}, \mathrm{H}_{2} \mathrm{O}$ was added to the reaction mixture to quench the reaction and the reaction mixture was extracted with dichloromethane. The organic layers were combined, dried over $\mathrm{MgSO}_{4}$, and concentrated. The crude mixture was purified by flash column chromatography on silica gel using a 1:3 mixture of ethyl acetate and hexanes as the eluent to provide the desired product $\mathbf{1 0 a}(1.7 \mathrm{~g}, 78 \%)$ as a brown solid.

${ }^{1} \mathbf{H}$ NMR $\left(500 \mathrm{MHz}, \mathrm{CDCl}_{3}, \mathrm{ppm}\right): \delta 8.37(\mathrm{~d}, J=5.5 \mathrm{~Hz}, 1 \mathrm{H}), 7.61(\mathrm{~d}, J=1.5 \mathrm{~Hz}, 1 \mathrm{H}), 7.43(\mathrm{dd}, J=$ 5.3, $1.7 \mathrm{~Hz}, 1 \mathrm{H}), 4.52(\mathrm{~d}, J=5.2 \mathrm{~Hz}, 2 \mathrm{H}), 2.80(\mathrm{br}, 1 \mathrm{H}) .{ }^{13} \mathbf{C} \mathbf{N M R}\left(125 \mathrm{MHz}, \mathrm{CDCl}_{3}, \mathrm{ppm}\right): \delta 150.5$, 143.9, 133.0, 130.4, 126.7, 89.4, 83.6, 51.3. IR (film): 3264, 2226, 1567, 1540, 1464, 1373, 1052 , 826, $686 \mathrm{~cm}^{-1}$; HRMS (ESI) calcd for $\mathrm{C}_{8} \mathrm{H}_{6} \mathrm{BrNONa}[\mathrm{M}+\mathrm{Na}]$ 233.9531, found 233.9538.

To confirm the structure of the desired product $10 \mathrm{a}$, compound $\mathbf{1 0 a}$ was further converted into its reduced analogue red-10a.

\section{3-(Pyridin-2-yl)propan-1-ol (red-10a)}

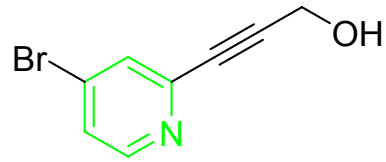

$10 a$

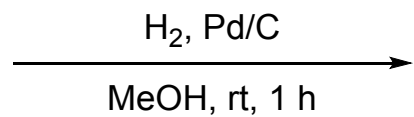

$\mathrm{MeOH}, \mathrm{rt}, 1 \mathrm{~h}$

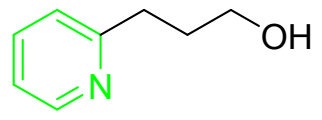

red-10a

To a solution of $10 \mathrm{a}(21 \mathrm{mg}, 0.10 \mathrm{mmol})$ in methanol $(1.0 \mathrm{~mL})$ was added $\mathrm{Pd} / \mathrm{C}(21 \mathrm{mg})$. The mixture was stirred at room temperature under $1.0 \mathrm{~atm}$ of hydrogen atmosphere and monitored by TLC. After $1 \mathrm{~h}$, the reaction mixture was filtered through celite to remove the undissolved solid. The filtrate was concentrated and purified by flash column chromatography on silica gel using a 1:1 mixture of ethyl acetate and hexanes as the eluent to afford compound red-10a (13 mg, 92\%) as yellow oil. Spectroscopic data was in good match with one obtained from a commercial source. ${ }^{\mathbf{1}} \mathbf{H}$ NMR $\left(500 \mathrm{MHz}, \mathrm{CDCl}_{3}, \mathrm{ppm}\right): \delta 8.50(\mathrm{~d}, J=4.6 \mathrm{~Hz}, 1 \mathrm{H}), 7.61(\mathrm{td}, J=7.6,1.5 \mathrm{~Hz}, 1 \mathrm{H}), 7.19(\mathrm{~d}, J$ 
$=7.6 \mathrm{~Hz}, 1 \mathrm{H}), 7.13(\mathrm{dd}, J=7.0,5.2 \mathrm{~Hz}, 1 \mathrm{H}), 4.12(\mathrm{br}, 1 \mathrm{H}), 3.72(\mathrm{t}, J=5.8 \mathrm{~Hz}, 2 \mathrm{H}), 2.98(\mathrm{t}, J=6.7$ $\mathrm{Hz}, 2 \mathrm{H}), 1.96-2.04(\mathrm{~m}, 2 \mathrm{H})$.

Based on this ${ }^{1} \mathrm{H}$ NMR result, we reached the conclusion that the Sonogashira coupling reaction would take place at the bromide at the 2-position of rather than one at the 4-position.

\section{2-3. 3-(5-Bromopyridin-2-yl)prop-2-yn-1-ol (10b)}<smiles>Brc1ccc(Br)nc1</smiles>

11b

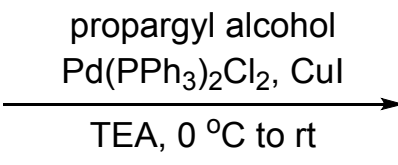

TEA, $0{ }^{\circ} \mathrm{C}$ to rt

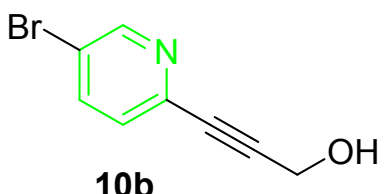

$10 \mathrm{~b}$

To a solution of compound $\mathbf{1 1 b}(2.4 \mathrm{~g}, 10 \mathrm{mmol}), \mathrm{Pd}\left(\mathrm{PPh}_{3}\right)_{2} \mathrm{Cl}_{2}(0.21 \mathrm{~g}, 0.30 \mathrm{mmol})$ and copper(I) iodide $(57 \mathrm{mg}, 0.30 \mathrm{mmol})$ in triethylamine $(20 \mathrm{~mL})$ was added propargyl alcohol $(0.58 \mathrm{~mL}, 10$ mmol). The above mixture was stirred at $0{ }^{\circ} \mathrm{C}$ under nitrogen atmosphere for $10 \mathrm{~min}$, then warmed up to room temperature and stirred for $2 \mathrm{~h}$. After complete consumption of $\mathbf{1 1 b}$, the reaction mixture was quenched by $\mathrm{H}_{2} \mathrm{O}$, and extracted with dichloromethane. The organic layers were combined, dried over $\mathrm{MgSO}_{4}$, and concentrated. The residue was purified by flash column chromatography on silica gel using a 1:3 mixture of ethyl acetate and hexanes as the eluent to afford compound $\mathbf{1 0 b}(1.8$ $\mathrm{g}, 83 \%$ ) as a white solid. Spectroscopic data was in good match with a reported value in the literature. ${ }^{2}{ }^{1} \mathbf{H}$ NMR $\left(500 \mathrm{MHz}, \mathrm{CDCl}_{3}, \mathrm{ppm}\right): \delta 8.62(\mathrm{~s}, 1 \mathrm{H}), 7.78(\mathrm{dd}, J=8.4,2.0 \mathrm{~Hz}, 1 \mathrm{H}), 7.31(\mathrm{~d}$, $J=8.2 \mathrm{~Hz}, 1 \mathrm{H}), 4.51(\mathrm{~s}, 2 \mathrm{H}), 3.27(\mathrm{br}, 1 \mathrm{H})$. 


\section{Total Synthesis of Phenanthroindolizidine Alkaloids}

\section{3-1. Total Synthesis of Natural Products in Group A}

\section{Synthesis of Compound 13}
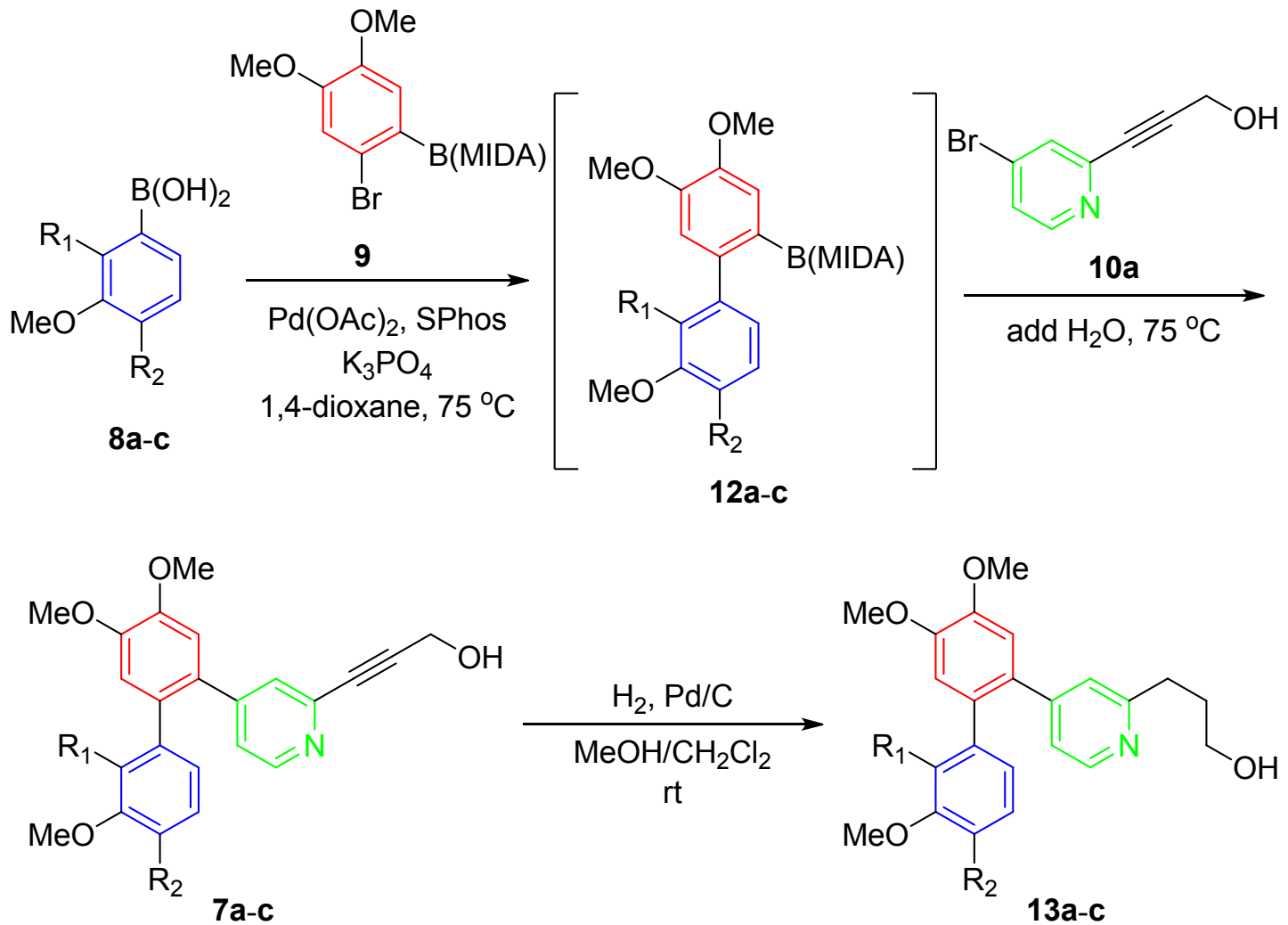

In a one-neck round bottom flask were placed 8 (2.4 mmol), $9(0.74 \mathrm{~g}, 2.0 \mathrm{mmol}), \mathrm{Pd}(\mathrm{OAc})_{2}(45 \mathrm{mg}$, $0.20 \mathrm{~mol})$, SPhos $(0.16 \mathrm{~g}, 0.40 \mathrm{mmol})$, and $\mathrm{K}_{3} \mathrm{PO}_{4}(1.3 \mathrm{~g}, 6.0 \mathrm{mmol})$ under argon atmosphere. To the flask was added $20 \mathrm{~mL}$ of 1,4-dioxane and the reaction mixture was heated to $75^{\circ} \mathrm{C}$ and monitored by TLC. After $3 \mathrm{~h}, \mathbf{1 0 a}(0.51 \mathrm{~g}, 2.4 \mathrm{mmol})$ and water $(4.0 \mathrm{~mL})$ were directly added to the reaction mixture and the reaction mixture was further stirred for $3 \mathrm{~h}$. After $3 \mathrm{~h}$, the reaction mixture was cooled to room temperature, quenched with water $(20 \mathrm{~mL})$ and extracted with dichloromethane. The combined organic layers were washed with brine, dried over $\mathrm{MgSO}_{4}$ and concentrated under reduced pressure. Without further purification, the crude mixture was re-dissolved in a mixture of methanol and dichloromethane $(2: 1,20 \mathrm{~mL})$ and $\mathrm{Pd} / \mathrm{C}$ powder $(0.40 \mathrm{~g})$ was added to the solution. The mixture was stirred at room temperature under $1.0 \mathrm{~atm}$ of hydrogen. After $3 \mathrm{~h}$, the mixture was filtered to remove insoluble solid. The filtrate was concentrated and purified by flash column chromatography on silica gel using a 1:1 mixture of tetrahydrofuran and hexanes as the eluent to afford compound $\mathbf{1 3 .}$ 


\section{Compound 13a}<smiles>COc1ccc(-c2cc(OC)c(OC)cc2-c2ccnc(CCCO)c2)cc1OC</smiles>

A white solid; $0.51 \mathrm{~g}, 62 \%$ yield. ${ }^{1} \mathbf{H}$ NMR $\left(500 \mathrm{MHz}, \mathrm{CDCl}_{3}, \mathrm{ppm}\right): \delta 8.35(\mathrm{~d}, J=4.9 \mathrm{~Hz}, 1 \mathrm{H}), 6.95$ (s, 2H), $6.91(\mathrm{~d}, J=10.1 \mathrm{~Hz}, 2 \mathrm{H}), 6.76-6.81(\mathrm{~m}, 1 \mathrm{H}), 6.69-6.75(\mathrm{~m}, 1 \mathrm{H}), 6.54(\mathrm{~s}, 1 \mathrm{H}), 3.96(\mathrm{~s}, 6 \mathrm{H})$, $3.87(\mathrm{~s}, 3 \mathrm{H}), 3.62(\mathrm{~s}, 3 \mathrm{H}), 3.59(\mathrm{t}, J=5.8 \mathrm{~Hz}, 2 \mathrm{H}), 2.83(\mathrm{t}, J=6.7 \mathrm{~Hz}, 2 \mathrm{H}), 1.81-1.88(\mathrm{~m}, 2 \mathrm{H}) ;{ }^{13} \mathrm{C}$ NMR (125 MHz, $\left.\mathrm{CDCl}_{3}, \mathrm{ppm}\right): \delta 161.1,150.6,149.3,148.5,148.5,148.2,133.4,130.1,124.5$, 122.4, 122.2, 113.7, 113.6, 113.0, 111.1, 62.3, 56.4, 56.3, 56.1, 55.9, 35.4, 31.7; IR (film): 3309, 2928, 2852, 1602, 1507, 1250, 1025, $801 \mathrm{~cm}^{-1}$; HRMS (ESI) calcd for $\mathrm{C}_{24} \mathrm{H}_{27} \mathrm{NO}_{5} \mathrm{Na}[\mathrm{M}+\mathrm{Na}]$ 432.1787, found 432.1784 .

\section{Compound 13b}<smiles>COc1cccc(-c2cc(OC)c(OC)cc2-c2ccnc(CCCO)c2)c1</smiles>

A white solid; $0.36 \mathrm{~g}, 47 \%$ yield. ${ }^{1} \mathbf{H}$ NMR $\left(500 \mathrm{MHz}, \mathrm{CDCl}_{3}, \mathrm{ppm}\right): \delta 8.32(\mathrm{~d}, J=5.2 \mathrm{~Hz}, 1 \mathrm{H}), 7.15$ (t, $J=7.9 \mathrm{~Hz}, 1 \mathrm{H}), 6.94(\mathrm{~s}, 2 \mathrm{H}), 6.90(\mathrm{~d}, J=5.5 \mathrm{~Hz}, 2 \mathrm{H}), 6.77(\mathrm{dd}, J=8.1,1.7 \mathrm{~Hz}, 1 \mathrm{H}), 6.70(\mathrm{~d}, J=$ $7.6 \mathrm{~Hz}, 1 \mathrm{H}), 6.64(\mathrm{~s}, 1 \mathrm{H}), 3.95(\mathrm{~s}, 3 \mathrm{H}), 3.94(\mathrm{~s}, 3 \mathrm{H}), 3.66(\mathrm{~s}, 3 \mathrm{H}), 3.57(\mathrm{t}, J=5.8 \mathrm{~Hz}, 2 \mathrm{H}) ;{ }^{13} \mathbf{C ~ N M R}$ (125 MHz, $\left.\mathrm{CDCl}_{3}, \mathrm{ppm}\right): \delta 161.0,159.3,150.2,149.1,148.6,148.4,142.2,133.4,130.1,129.3$, $124.4,122.5,122.3,115.7,113.7,112.9,112.4,62.1,56.3,56.2,55.2,35.2,31.7$; IR (film): 3311 , 2935, 2852, 1602, 1517, 1478, 1248, 1204, 1155, 1031, $774 \mathrm{~cm}^{-1}$; HRMS (ESI) calcd for $\mathrm{C}_{23} \mathrm{H}_{25} \mathrm{NO}_{4} \mathrm{Na}[\mathrm{M}+\mathrm{Na}]$ 402.1681, found 402.1678. 


\section{Compound 13c}

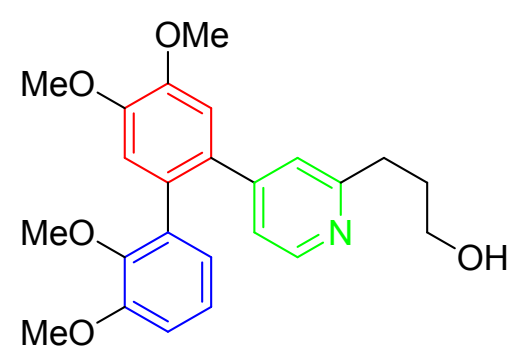

A white solid; $0.39 \mathrm{~g}, 24 \%$ yield. ${ }^{1} \mathbf{H}$ NMR (500 MHz, $\left.\mathrm{CDCl}_{3}, \mathrm{ppm}\right): \delta 8.29(\mathrm{~d}, J=4.9 \mathrm{~Hz}, 1 \mathrm{H}), 6.89$ - $6.98(\mathrm{~m}, 5 \mathrm{H}), 6.84(\mathrm{~d}, J=8.2 \mathrm{~Hz}, 1 \mathrm{H}), 6.62(\mathrm{~d}, J=7.6 \mathrm{~Hz}, 1 \mathrm{H}), 3.95(\mathrm{~s}, 3 \mathrm{H}), 3.91(\mathrm{~s}, 3 \mathrm{H}), 3.82(\mathrm{~s}$, $3 \mathrm{H}), 3.54(\mathrm{t}, J=5.7 \mathrm{~Hz}, 2 \mathrm{H}), 3.50(\mathrm{~s}, 3 \mathrm{H}), 2.81(\mathrm{t}, J=6.7 \mathrm{~Hz}, 2 \mathrm{H}), 1.77-1.86(\mathrm{~m}, 2 \mathrm{H}) ;{ }^{13} \mathbf{C} \mathbf{N M R}$ (125 MHz, $\left.\mathrm{CDCl}_{3}, \mathrm{ppm}\right): \delta 160.8,152.9,150.3,148.8,148.6,148.3,146.6,134.9,130.9,129.6$, 123.9, 123.8, 123.6, 121.8, 114.3, 112.4, 111.8, 62.1, 60.4, 56.2, 56.2, 55.9, 35.2, 31.6; IR (film): 3303, 2924, 2852, 1507, 1462, 1248, 1174, 1025, 859, $764 \mathrm{~cm}^{-1}$; HRMS (ESI) calcd for $\mathrm{C}_{24} \mathrm{H}_{27} \mathrm{NO}_{5} \mathrm{Na}[\mathrm{M}+\mathrm{Na}]$ 432.1787, found 432.1782. 
<smiles>[R2]c1ccc(-c2cc(OC)c(OC)cc2-c2ccnc(CCCO)c2)c([R])c1OC</smiles>

To a solution of $13(1.0 \mathrm{mmol})$ in dichloromethane $(10 \mathrm{~mL})$ were added $\mathrm{MsCl}(0.15 \mathrm{~mL}, 2.0 \mathrm{mmol})$ and TEA $(0.41 \mathrm{~mL}, 3.0 \mathrm{mmol})$ at $0{ }^{\circ} \mathrm{C}$. The reaction mixture was warmed up to room temperature and stirred for $2 \mathrm{~h}$. After $2 \mathrm{~h}$, the reaction mixture was concentrated under reduced pressure. recrystallization in a mixture of dichloromethane and ethyl acetate provided the crude mixture of compound 6, which was directly used for the next step. To a solution of the resulting solid in methanol $(10 \mathrm{~mL})$ was added $\mathrm{NaBH}_{4}(0.15 \mathrm{~g}, 4.0 \mathrm{mmol})$ and the reaction mixture was stirred at room temperature. After $30 \mathrm{~min}$, the reaction mixture was quenched with water $(10 \mathrm{~mL})$ and extracted with ethyl acetate. The organic layers were combined, washed with brine, dried over $\mathrm{MgSO}_{4}$ and concentrated under reduced pressure. The residue was purified by column chromatography on silica gel using a 10:1 mixture of dichloromethane and methanol as the eluent to provide the product 14 .

\section{Compound 14a}<smiles>COc1ccc(-c2cc(OC)c(OC)cc2C2=CCN3CCCC3C2)cc1OC</smiles>

A white solid; $0.32 \mathrm{~g}, 81 \%$ yield. Spectroscopic data was in good match with a reported value in the literature. ${ }^{3}{ }^{1} \mathbf{H}$ NMR (500 MHz, $\left.\mathrm{CDCl}_{3}, \mathrm{ppm}\right): \delta 6.97(\mathrm{~s}, 1 \mathrm{H}), 6.94(\mathrm{~d}, J=8.2 \mathrm{~Hz}, 1 \mathrm{H}), 6.89(\mathrm{~d}, J=$ $8.2 \mathrm{~Hz}, 1 \mathrm{H}), 6.82(\mathrm{~s}, 1 \mathrm{H}), 6.81(\mathrm{~s}, 1 \mathrm{H}), 5.74(\mathrm{~d}, J=2.8 \mathrm{~Hz}, 1 \mathrm{H}), 3.92(\mathrm{~s}, 3 \mathrm{H}), 3.89$ (s, 3H), 3.89 (s, 
3H), $3.88(\mathrm{~s}, 3 \mathrm{H}), 3.67(\mathrm{~d}, J=14.7 \mathrm{~Hz}, 1 \mathrm{H}), 3.25(\mathrm{~m}, 1 \mathrm{H}), 2.93(\mathrm{~d}, J=13.4 \mathrm{~Hz}, 1 \mathrm{H}), 2.17$ - 2.27 (m, 1H), $2.12(\mathrm{~m}, 1 \mathrm{H}), 1.98(\mathrm{~m}, 2 \mathrm{H}), 1.85(\mathrm{~m}, 2 \mathrm{H}), 1.73(\mathrm{~m}, 1 \mathrm{H}), 1.37(\mathrm{~m}, 1 \mathrm{H})$.

\section{Compound 14b}<smiles>COc1cccc(-c2cc(OC)c(OC)cc2C2=CCN3CCCC3C2)c1</smiles>

A white solid; $0.31 \mathrm{~g}, 86 \%$ yield. Spectroscopic data was in good match with a reported value in the literature. ${ }^{3}{ }^{1} \mathbf{H}$ NMR (500 MHz, $\left.\mathrm{CDCl}_{3}, \mathrm{ppm}\right): \delta 7.25$ - 7.29 (t, $\left.J=7.8 \mathrm{~Hz}, 1 \mathrm{H}\right), 6.94-7.01(\mathrm{~m}, 2 \mathrm{H})$, $6.82-6.86(\mathrm{~m}, 2 \mathrm{H}), 6.82(\mathrm{~s}, 1 \mathrm{H}), 5.71(\mathrm{~d}, J=3.1 \mathrm{~Hz}, 1 \mathrm{H}), 3.89(\mathrm{~s}, 6 \mathrm{H}), 3.82(\mathrm{~s}, 3 \mathrm{H}), 3.57$ - $3.66(\mathrm{~m}$, 1H), $3.21(\mathrm{~m}, 1 \mathrm{H}), 2.89(\mathrm{~m}, 1 \mathrm{H}), 2.17(\mathrm{~m}, 1 \mathrm{H}), 1.90-2.11(\mathrm{~m}, 3 \mathrm{H}), 1.82(\mathrm{~m}, 2 \mathrm{H}), 1.71(\mathrm{~m}, 1 \mathrm{H})$, $1.29-1.37(\mathrm{~m}, 1 \mathrm{H})$.

\section{Compound 14c}

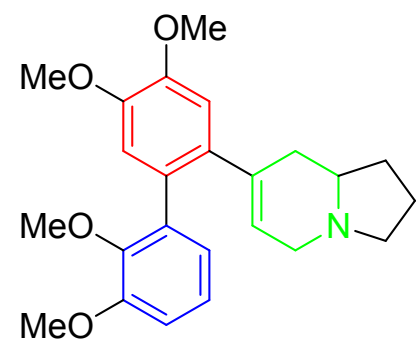

A white solid; $0.33 \mathrm{~g}, 84 \%$ yield. Spectroscopic data was in good match with a reported value in the literature. ${ }^{3}{ }^{1} \mathbf{H}$ NMR $\left(500 \mathrm{MHz}, \mathrm{CDCl}_{3}, \mathrm{ppm}\right): \delta 7.01$ (t, $\left.J=7.9,1 \mathrm{H}\right), 6.87(\mathrm{~d}, J=7.9,1 \mathrm{H}), 6.86$ (s, 1H), $6.82(\mathrm{~d}, J=7.9,1 \mathrm{H}), 6.82(\mathrm{~s}, 1 \mathrm{H}), 5.50(\mathrm{br}, 1 \mathrm{H}), 3.89(\mathrm{~s}, 6 \mathrm{H}), 3.85(\mathrm{~s}, 3 \mathrm{H}), 3.58(\mathrm{~s}, 3 \mathrm{H}), 3.53(\mathrm{~d}$, $J=16.2 \mathrm{~Hz}, 1 \mathrm{H}), 3.19(\mathrm{br}, 1 \mathrm{H}), 2.80(\mathrm{br}, 1 \mathrm{H}), 2.04-2.28(\mathrm{~m}, 4 \mathrm{H}), 1.80-1.95(\mathrm{~m}, 2 \mathrm{H}), 1.73(\mathrm{br}, 1 \mathrm{H})$, $1.42(\mathrm{br}, 1 \mathrm{H})$. 


\section{Synthesis of Natural Products in Group A}<smiles>[R2]c1ccc(-c2cc(OC)c(OC)cc2C2=CCN3CCCC3C2)c([R])c1OC</smiles>

$14 a-c$

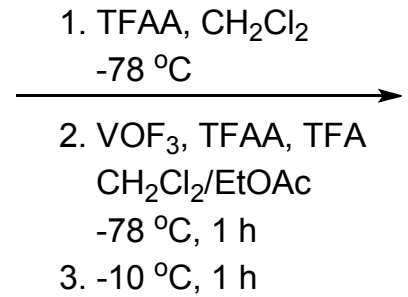

3. $-10^{\circ} \mathrm{C}, 1 \mathrm{~h}$

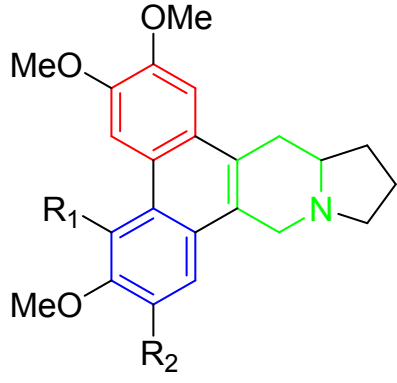

1-3

Preparation of $\mathrm{VOF}_{3}$ solution : To a solution of the $\mathrm{VOF}_{3}(1.0 \mathrm{~g}, 8.0 \mathrm{mmol})$ in anhydrous dichloromethane $(20 \mathrm{~mL})$ and anhydrous ethyl acetate $(10 \mathrm{~mL})$ were added trifluoroacetic acid (TFA, $1.0 \mathrm{~mL}, 13 \mathrm{mmol}$ ) and trifluoroacetic anhydride (TFAA, 4 drops) under nitrogen atmosphere and the mixture was stirred at room temperature.

A solution of $14(0.50 \mathrm{mmol})$ and TFAA (2 drops) in anhydrous dichloromethane $(100 \mathrm{~mL})$ was stirred at $-78{ }^{\circ} \mathrm{C}$ under nitrogen atmosphere. To the solution was added the prepared $\mathrm{VOF}_{3}$ solution $(4.9 \mathrm{~mL}, 1.3 \mathrm{mmol})$ over 10 minutes. After $1 \mathrm{~h}$, the reaction mixture was warmed to $-10{ }^{\circ} \mathrm{C}$ and further stirred for additional $1 \mathrm{~h}$ at the same temperature. After $1 \mathrm{~h}, 50 \mathrm{~mL}$ of $10 \% \mathrm{NaOH}$ solution was directly added to the reaction mixture and the reaction mixture was vigorously stirred for $1 \mathrm{~h}$ at room temperature. Then, the reaction mixture was extracted with dichloromethane, and the organic layers were combined, dried over $\mathrm{MgSO}_{4}$, and concentrated. The crude mixture was purified by flash column chromatography on silica gel using a 10:1 mixture of dichloromethane and methanol as the eluent to provide the natural product in group A.

\section{Tylophorine (1)}<smiles>COc1cc2c3c(c4cc(OC)c(OC)cc4c2cc1OC)CN1CCCC1C3</smiles> 
A yellow solid; $0.16 \mathrm{~g}, 82 \%$ yield. Spectroscopic data was in good match with a reported value in the literature. ${ }^{3}{ }^{1} \mathbf{H}$ NMR (500 MHz, $\left.\mathrm{CDCl}_{3}, \mathrm{ppm}\right): \delta 7.83(\mathrm{~s}, 1 \mathrm{H}), 7.83(\mathrm{~s}, 1 \mathrm{H}), 7.32(\mathrm{~s}, 1 \mathrm{H}), 7.16$ (s, 1H), $4.63(\mathrm{~d}, J=14.7 \mathrm{~Hz}, 1 \mathrm{H}), 4.12(\mathrm{~s}, 6 \mathrm{H}), 4.06(\mathrm{~s}, 3 \mathrm{H}), 4.05(\mathrm{~s}, 3 \mathrm{H}), 3.68(\mathrm{~d}, J=14.3 \mathrm{~Hz}, 1 \mathrm{H})$, $3.49(\mathrm{t}, J=7.9 \mathrm{~Hz}, 1 \mathrm{H}), 3.38(\mathrm{dd}, J=15.9,2.4 \mathrm{~Hz}, 1 \mathrm{H}), 2.87-2.97(\mathrm{~m}, 1 \mathrm{H}), 2.43-2.56(\mathrm{~m}, 2 \mathrm{H})$, $2.22-2.30(\mathrm{~m}, 1 \mathrm{H}), 2.05(\mathrm{dd}, J=11.6,8.6 \mathrm{~Hz}, 1 \mathrm{H}), 1.89-1.98(\mathrm{~m}, 1 \mathrm{H}), 1.74-1.83(\mathrm{~m}, 1 \mathrm{H}) ;{ }^{13} \mathrm{C}$ NMR (125 MHz, $\left.\mathrm{CDCl}_{3}, \mathrm{ppm}\right): \delta 148.9,148.7,148.6,126.5,126.0,124.5,123.8,123.6,104.1$, 103.6, 103.5, 103.3, 60.4, 56.2, 56.1, 56.0, 55.3, 54.2, 34.0, 31.4, 21.8; IR (film): 2922, 2852, 1619, 1514, 1470, 1427, 1248, 1213, 1151, 1017, 842, $750 \mathrm{~cm}^{-1}$; HRMS (ESI) calcd for $\mathrm{C}_{24} \mathrm{H}_{27} \mathrm{NO}_{4} \mathrm{Na}$ $[\mathrm{M}+\mathrm{Na}]$ 416.1838, found 416.1835.

\section{Antofine (2)}

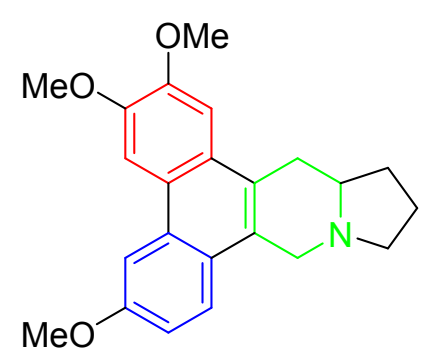

A yellow solid; $0.14 \mathrm{~g}, 79 \%$ yield. Spectroscopic data was in good match with a reported value in the literature. ${ }^{3}{ }^{1} \mathbf{H}$ NMR $\left(500 \mathrm{MHz}, \mathrm{CDCl}_{3}, \mathrm{ppm}\right): \delta 7.90(\mathrm{~s}, 1 \mathrm{H}), 7.89(\mathrm{~d}, J=2.1 \mathrm{~Hz}, 1 \mathrm{H}), 7.81(\mathrm{~d}, J=$ $8.9 \mathrm{~Hz}, 1 \mathrm{H}), 7.30(\mathrm{~s}, 1 \mathrm{H}), 7.20(\mathrm{dd}, J=8.9,2.1 \mathrm{~Hz}, 1 \mathrm{H}), 4.69(\mathrm{~d}, J=14.7 \mathrm{~Hz}, 1 \mathrm{H}), 4.10(\mathrm{~s}, 3 \mathrm{H}), 4.06$ $(\mathrm{s}, 3 \mathrm{H}), 4.01(\mathrm{~s}, 3 \mathrm{H}), 3.69(\mathrm{~d}, J=15.0 \mathrm{~Hz}, 1 \mathrm{H}), 3.46(\mathrm{t}, J=8.1 \mathrm{~Hz}, 1 \mathrm{H}), 3.33(\mathrm{dd}, J=15.6,2.1 \mathrm{~Hz}$, 1H), $2.85-2.94(\mathrm{~m}, 1 \mathrm{H}), 2.41-2.55(\mathrm{~m}, 2 \mathrm{H}), 2.20-2.28(\mathrm{~m}, 1 \mathrm{H}), 1.99-2.08(\mathrm{~m}, 1 \mathrm{H}), 1.88-1.96$ (m, 1H), $1.73-1.82(\mathrm{~m}, 1 \mathrm{H}) ;{ }^{13} \mathbf{C}$ NMR (125 MHz, $\left.\mathrm{CDCl}_{3}, \mathrm{ppm}\right): \delta 157.6,149.5,148.5,130.3$, $127.2,126.7,125.7,124.4,124.2,123.7,115.0,104.8,104.1,103.9,60.4,56.1,56.0,55.7,55.2$, 53.9, 33.8, 31.4, 21.7; IR (film): 2916, 1615, 1512, 1468, 1256, 1205, 1126, 1042, 842, $735 \mathrm{~cm}^{-1}$; HRMS (ESI) calcd for $\mathrm{C}_{23} \mathrm{H}_{25} \mathrm{NO}_{3} \mathrm{Na}[\mathrm{M}+\mathrm{Na}]$ 386.1732, found 386.1735. 
Tylocrebrine (3)

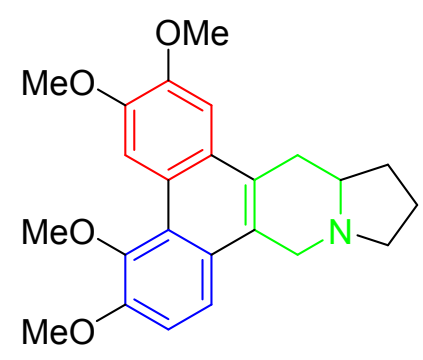

A yellow solid; $0.13 \mathrm{~g}, 65 \%$ yield. Spectroscopic data was in good match with a reported value in the literature. ${ }^{3}{ }^{1} \mathbf{H}$ NMR (500 MHz, $\left.\mathrm{CDCl}_{3}, \mathrm{ppm}\right): \delta 9.33(\mathrm{~s}, 1 \mathrm{H}), 7.66(\mathrm{~d}, J=9.2 \mathrm{~Hz}, 1 \mathrm{H}), 7.33(\mathrm{~s}, 1 \mathrm{H})$, $7.29(\mathrm{~d}, J=9.2 \mathrm{~Hz}, 1 \mathrm{H}), 4.67(\mathrm{~d}, J=15.0 \mathrm{~Hz}, 1 \mathrm{H}), 4.07$ (s, 6H), $4.04(\mathrm{~s}, 3 \mathrm{H}), 3.92(\mathrm{~s}, 3 \mathrm{H}), 3.69$ (d, $\mathrm{J}=14.7 \mathrm{~Hz}, 1 \mathrm{H}), 3.44-3.50(\mathrm{~m}, 1 \mathrm{H}), 3.34(\mathrm{dd}, J=15.7,2.3 \mathrm{~Hz}, 1 \mathrm{H}), 2.88-2.97(\mathrm{~m}, 1 \mathrm{H}), 2.43-2.54$ (m, 2H), $2.21-2.29(\mathrm{~m}, 1 \mathrm{H}), 2.00-2.08(\mathrm{~m}, 1 \mathrm{H}), 1.88-1.98(\mathrm{~m}, 1 \mathrm{H}), 1.75-1.84(\mathrm{~m}, 1 \mathrm{H}) ;{ }^{13} \mathrm{C}$ NMR (125 MHz, $\left.\mathrm{CDCl}_{3}, \mathrm{ppm}\right): \delta$ 150.7, 148.8, 147.9, 146.4, 126.6, 125.9, 123.7, 123.4, 119.0, 112.2, 109.3, 103.6, 60.4, 60.2, 56.6, 55.9, 55.3, 54.4, 34.1, 31.4, 21.8; IR (film): 2928, 1717, 1569, 1445, 1255, 1182, 1114, 1027, 823, $739 \mathrm{~cm}^{-1}$; HRMS (ESI) calcd for $\mathrm{C}_{24} \mathrm{H}_{27} \mathrm{NO}_{4} \mathrm{Na}[\mathrm{M}+\mathrm{Na}]$ 416.1838, found 416.1833. 


\section{3-2. Total Synthesis of Natural Products in Group B}

\section{Synthesis of Compound 13}

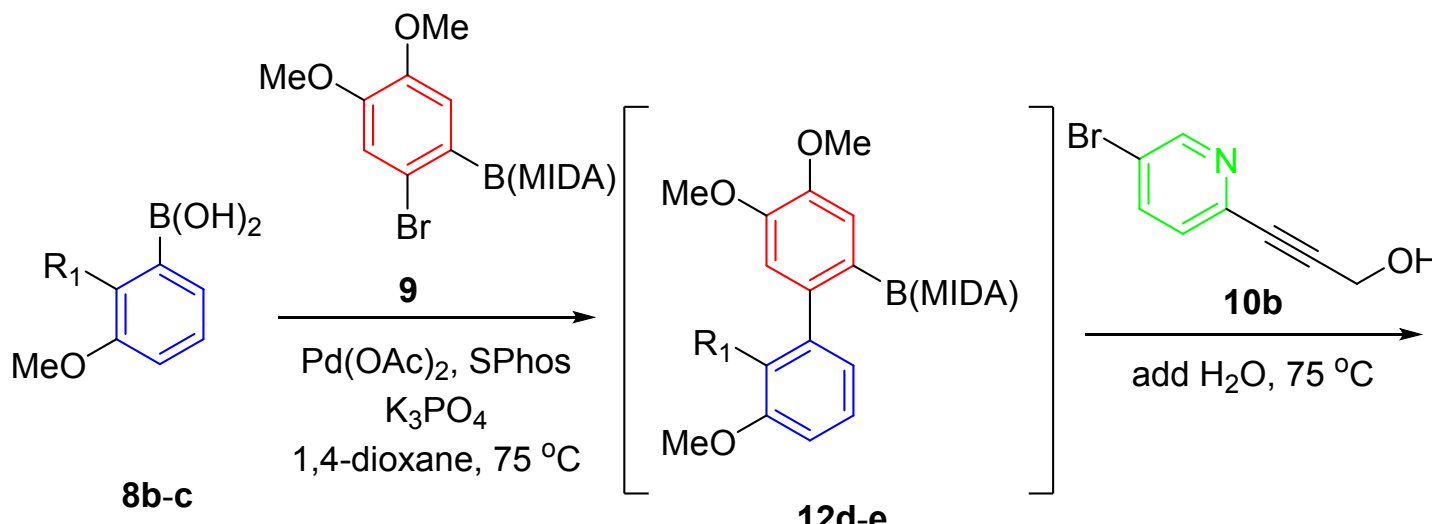

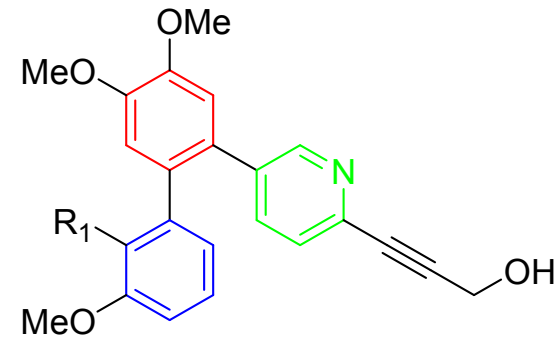

7d-e

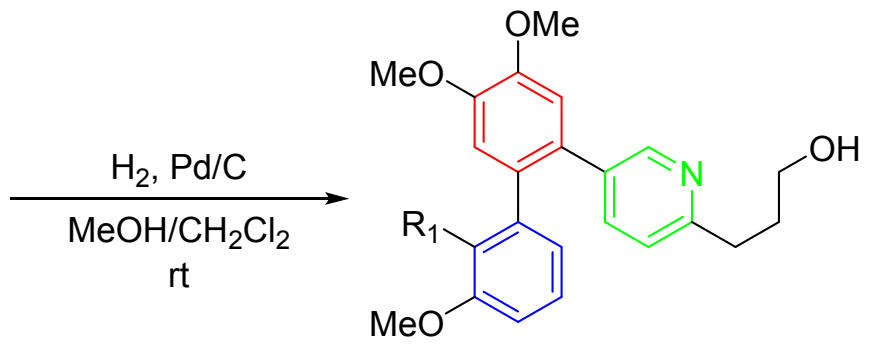

$13 d-e$

In a one-neck round bottom flask were placed 8 (2.4 mmol), $9(0.74 \mathrm{~g}, 2.0 \mathrm{mmol}), \mathrm{Pd}(\mathrm{OAc})_{2}(45 \mathrm{mg}$, $0.20 \mathrm{~mol})$, SPhos $(0.16 \mathrm{~g}, 0.40 \mathrm{mmol})$, and $\mathrm{K}_{3} \mathrm{PO}_{4}(1.3 \mathrm{~g}, 6.0 \mathrm{mmol})$ under argon atmosphere. To the flask was added 1,4-dioxane $(20 \mathrm{~mL})$ and the reaction mixture was heated to $75{ }^{\circ} \mathrm{C}$ and was stirred for $3 \mathrm{~h}$. After $3 \mathrm{~h}, \mathbf{1 0 b}(0.51 \mathrm{~g}, 2.4 \mathrm{mmol})$ and $4.0 \mathrm{~mL}$ of water were directly added to the reaction mixture and the reaction mixture was further stirred at the same temperature. After $3 \mathrm{~h}$, the reaction mixture was cooled to room temperature, quenched with water $(20 \mathrm{~mL})$ and extracted with dichloromethane. The combined organic layers were washed with brine, dried over $\mathrm{MgSO}_{4}$ and concentrated under reduced pressure. Without further purification, the crude mixture was redissolved in a mixture of methanol and dichloromethane $(2: 1,20 \mathrm{~mL})$ and $\mathrm{Pd} / \mathrm{C}$ powder $(0.40 \mathrm{~g})$ was added to the solution. The mixture was stirred at room temperature under 1.0 atm of hydrogen for 3 $\mathrm{h}$ and then filtered to remove insoluble solid. The filtrate was concentrated and purified by flash column chromatography on silica gel using a 1:1 mixture of tetrahydrofuran and hexanes as the eluent to afford compound $\mathbf{1 3 .}$ 


\section{Compound 13d}

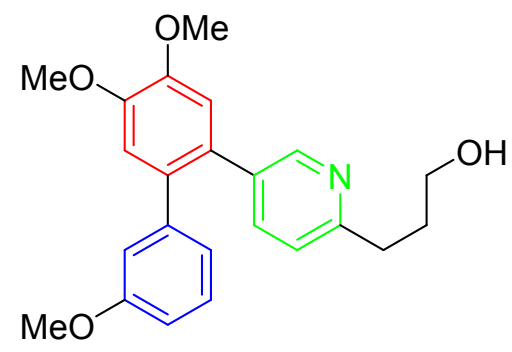

A white solid; $0.34 \mathrm{~g}, 45 \%$ yield. ${ }^{1} \mathbf{H}$ NMR $\left(500 \mathrm{MHz}, \mathrm{CDCl}_{3}, \mathrm{ppm}\right): \delta 8.39$ (s, 1H), 7.37 (d, $J=7.5$ Hz, 1H), 7.15 (t, $J=7.9 \mathrm{~Hz}, 1 \mathrm{H}), 7.05$ (d, $J=7.8 \mathrm{~Hz}, 1 \mathrm{H}), 6.96(\mathrm{~s}, 1 \mathrm{H}), 6.89$ (s, 1H), $6.77(\mathrm{dd}, J=$ 7.9, $2.1 \mathrm{~Hz}, 1 \mathrm{H}), 6.68(\mathrm{~d}, J=7.5 \mathrm{~Hz}, 1 \mathrm{H}), 6.66(\mathrm{~d}, J=2.3 \mathrm{~Hz}, 1 \mathrm{H}), 3.96(\mathrm{~s}, 3 \mathrm{H}), 3.95(\mathrm{~s}, 3 \mathrm{H}), 3.70(\mathrm{t}$, $J=5.7 \mathrm{~Hz}, 2 \mathrm{H}), 3.68(\mathrm{~s}, 3 \mathrm{H}), 2.99(\mathrm{t}, \mathrm{J}=6.4 \mathrm{~Hz}, 2 \mathrm{H}), 2.03(\mathrm{br}, 1 \mathrm{H}), 1.94-2.01(\mathrm{~m}, 2 \mathrm{H}) ;{ }^{13} \mathbf{C} \mathbf{N M R}$ (125 MHz, $\left.\mathrm{CDCl}_{3}, \mathrm{ppm}\right): \delta 159.4,159.2,149.2,148.8,148.7,142.3,138.1,134.8,133.5,129.3$, 129.0, 122.7, 122.3, 115.8, 113.8, 113.4, 112.5, 62.6, 56.3, 56.3, 55.3, 35.2, 31.5; IR (film): 3309, 2924, 2852, 1600, 1485, 1244, 1202, 1157, 1027, 856, 778, 733, $700 \mathrm{~cm}^{-1}$; HRMS (ESI) calcd for $\mathrm{C}_{23} \mathrm{H}_{25} \mathrm{NO}_{4} \mathrm{Na}[\mathrm{M}+\mathrm{Na}]$ 402.1681, found 402.1683.

\section{Compound 13e}

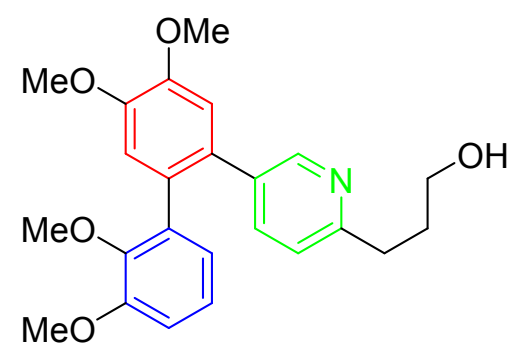

A white solid; $0.36 \mathrm{~g}, 22 \%$ yield. ${ }^{1} \mathbf{H}$ NMR $\left(500 \mathrm{MHz}, \mathrm{CDCl}_{3}, \mathrm{ppm}\right): \delta 8.32(\mathrm{~d}, J=1.8 \mathrm{~Hz}, 1 \mathrm{H}), 7.38$ $(\mathrm{dd}, J=8.1,2.3 \mathrm{~Hz}, 1 \mathrm{H}), 6.99(\mathrm{~d}, J=8.2 \mathrm{~Hz}, 1 \mathrm{H}), 6.94(\mathrm{~s}, 1 \mathrm{H}), 6.92(\mathrm{t}, J=7.9 \mathrm{~Hz}, 1 \mathrm{H}), 6.90(\mathrm{~s}, 1 \mathrm{H})$, $6.82(\mathrm{dd}, J=8.2,1.2 \mathrm{~Hz}, 1 \mathrm{H}), 6.61(\mathrm{dd}, J=7.6,1.2 \mathrm{~Hz}, 1 \mathrm{H}), 4.12(\mathrm{br}, 1 \mathrm{H}), 3.95(\mathrm{~s}, 3 \mathrm{H}), 3.91$ (s, 3H), $3.82(\mathrm{~s}, 3 \mathrm{H}), 3.67(\mathrm{t}, J=5.8 \mathrm{~Hz}, 2 \mathrm{H}), 3.50(\mathrm{~s}, 3 \mathrm{H}), 2.90(\mathrm{t}, J=6.7 \mathrm{~Hz}, 2 \mathrm{H}), 1.90-1.97(\mathrm{~m}, 2 \mathrm{H}) ;{ }^{13} \mathrm{C}$ NMR (125 MHz, $\left.\mathrm{CDCl}_{3}, \mathrm{ppm}\right): \delta$ 159.0, 153.0, 148.9, 148.6, 148.4, 146.7, 137.4, 135.0, 134.9, $129.9,129.6,124.1,123.7,122.3,114.4,112.9,111.7,62.6,60.4,56.2,55.9,35.3,31.5$; IR (film): 3322, 2926, 2850, 1520, 1468, 1260, 1241, 1110, 1017, 801, $768 \mathrm{~cm}^{-1}$; HRMS (ESI) calcd for $\mathrm{C}_{24} \mathrm{H}_{27} \mathrm{NO}_{5} \mathrm{Na}$ $[\mathrm{M}+\mathrm{Na}]$ 
<smiles>[R]c1cc(OC)c(OC)cc1-c1ccc(CCCO)nc1</smiles>

13d-e

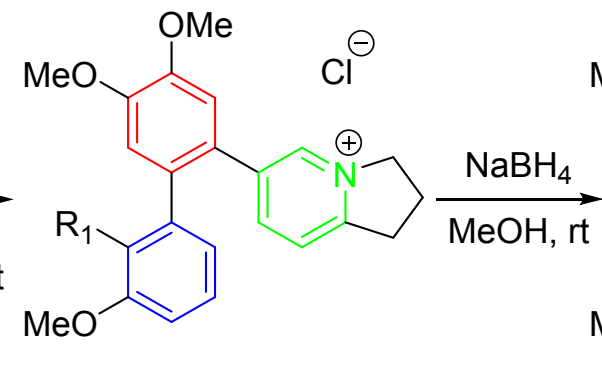

6d-e<smiles>[R]c1c(OC)cccc1-c1cc(OC)c(OC)cc1C1=CCC2CCCN2C1</smiles>

14d-e

To a solution of $13(1.0 \mathrm{mmol})$ in dichloromethane $(10 \mathrm{~mL})$ were added $\mathrm{MsCl}(0.15 \mathrm{~mL}, 2.0 \mathrm{mmol})$ and TEA $(0.41 \mathrm{~mL}, 3.0 \mathrm{mmol})$ at $0{ }^{\circ} \mathrm{C}$. The reaction mixture was warmed up to room temperature and stirred for $2 \mathrm{~h}$. After $2 \mathrm{~h}$, the reaction mixture was concentrated under reduced pressure to provide a crude product of compound $\mathbf{6}$. Recrystallization of $\mathbf{6}$ in a mixture of dichloromethane and ethyl acetate afforded the crude product of 6 as a yellow solid. To a solution of the resulting solid in methanol $(10 \mathrm{~mL})$ was added $\mathrm{NaBH}_{4}(0.15 \mathrm{~g}, 4.0 \mathrm{mmol})$ and the reaction mixture was stirred at room temperature. After $30 \mathrm{~min}$, the reaction mixture was quenched with water $(10 \mathrm{~mL})$ and extracted with ethyl acetate. The combined organic layers were washed with brine, dried over $\mathrm{MgSO}_{4}$ and concentrated under reduced pressure. The residue was purified by column chromatography on silica gel using a 10:1 mixture of dichloromethane and methanol as the eluent to provide the product 14 .

\section{Compound 14d}<smiles>COc1cccc(-c2cc(OC)c(OC)cc2C2=CCC3CCCN3C2)c1</smiles>

A white solid; $0.30 \mathrm{~g}, 82 \%$ yield. ${ }^{1} \mathbf{H}$ NMR $\left(500 \mathrm{MHz}, \mathrm{CDCl}_{3}, \mathrm{ppm}\right): \delta 7.23-7.28(\mathrm{t}, J=7.6 \mathrm{~Hz}$, 1H), $6.97(\mathrm{~d}, J=7.6 \mathrm{~Hz}, 1 \mathrm{H}), 6.95(\mathrm{~d}, J=1.8 \mathrm{~Hz}, 1 \mathrm{H}), 6.82-6.85(\mathrm{~m}, 1 \mathrm{H}), 6.82(\mathrm{~s}, 1 \mathrm{H}), 6.81(\mathrm{~s}, 1 \mathrm{H})$, $5.66(\mathrm{~d}, J=4.3 \mathrm{~Hz}, 1 \mathrm{H}), 3.89(\mathrm{~s}, 3 \mathrm{H}), 3.88(\mathrm{~s}, 3 \mathrm{H}), 3.81(\mathrm{~s}, 3 \mathrm{H}), 3.36(\mathrm{~d}, J=15.6 \mathrm{~Hz}, 1 \mathrm{H}), 3.07(\mathrm{t}, J$ $=8.1 \mathrm{~Hz}, 1 \mathrm{H}), 2.58(\mathrm{~d}, J=15.6 \mathrm{~Hz}, 1 \mathrm{H}), 2.24-2.34(\mathrm{~m}, 1 \mathrm{H}), 1.91-2.10(\mathrm{~m}, 4 \mathrm{H}), 1.77-1.87(\mathrm{~m}$, 
1H), $1.64-1.75(\mathrm{~m}, 1 \mathrm{H}), 1.35-1.47(\mathrm{~m}, 1 \mathrm{H}) ;{ }^{13} \mathbf{C}$ NMR (125 MHz, $\left.\mathrm{CDCl}_{3}, \mathrm{ppm}\right): \delta 159.3,148.0$, $147.9,143.3,137.5,133.1,132.5,129.1,125.8,121.9,114.9,113.2,112.8,112.3,59.2,56.3,56.1$, 56.1, 55.4, 54.3, 32.8, 30.6, 21.4; IR (film): 2925, 2854, 1598, 1505, 1462, 1378, 1256, 1208, 1173, 1025, $764 \mathrm{~cm}^{-1}$; HRMS (ESI) calcd for $\mathrm{C}_{23} \mathrm{H}_{25} \mathrm{NO}_{3} \mathrm{Na}$ [M+Na] 388.1889, found 388.1891.

\section{Compound 14e}

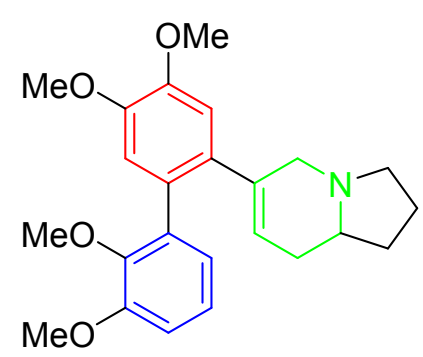

A white solid; 0.34 g, 87\% yield. ${ }^{1} \mathbf{H}$ NMR (500 MHz, $\left.\mathrm{CDCl}_{3}, \mathrm{ppm}\right): \delta 7.01$ (t, $\left.J=7.9 \mathrm{~Hz}, 1 \mathrm{H}\right), 6.79$ - $6.91(\mathrm{~m}, 4 \mathrm{H}), 5.54(\mathrm{br}, 1 \mathrm{H}), 3.90(\mathrm{~s}, 6 \mathrm{H}), 3.85(\mathrm{~s}, 3 \mathrm{H}), 3.58(\mathrm{~s}, 3 \mathrm{H}), 3.15(\mathrm{br}, 1 \mathrm{H}), 2.76(\mathrm{br}, 1 \mathrm{H})$, 1.82 - 2.25 (m, 7H), 1.74 (br, 1H), 1.45 (br, 1H); ${ }^{13}$ C NMR (125 MHz, $\left.\mathrm{CDCl}_{3}, \mathrm{ppm}\right): \delta 152.9,148.1$, $147.4,146.8,136.0,128.7,125.3,123.9,123.4,113.8,112.1,111.3,60.6,56.1,56.0,54.1,31.1$, 30.4, 21.3; IR (film): 2928, 2848, 1514, 1466, 1256, 1202, 1161, 1108, 1007, $766 \mathrm{~cm}^{-1}$; HRMS (ESI) calcd for $\mathrm{C}_{24} \mathrm{H}_{27} \mathrm{NO}_{4} \mathrm{Na}[\mathrm{M}+\mathrm{Na}] 418.1994$, found 418.1995. 
<smiles>[R]c1cc(OC)c(OC)cc1C1=CCC2CCCN2C1</smiles>

14d-e

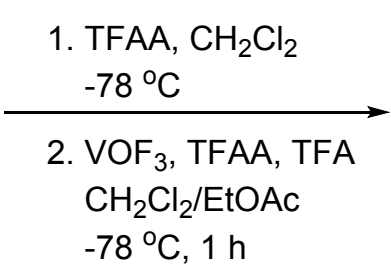

3. $-10^{\circ} \mathrm{C}, 1 \mathrm{~h}$

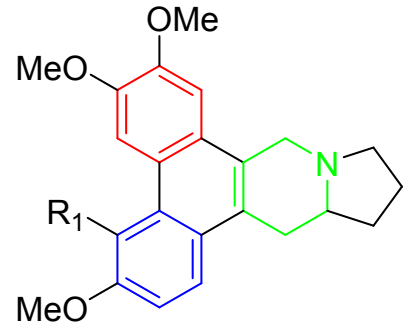

4-5

Preparation of $\mathrm{VOF}_{3}$ solution : To a solution of the $\mathrm{VOF}_{3}(1.0 \mathrm{~g}, 8.0 \mathrm{mmol})$ in anhydrous dichloromethane $(20 \mathrm{~mL})$ and anhydrous ethyl acetate $(10 \mathrm{~mL})$ were added trifluoroacetic acid (TFA, $1.0 \mathrm{~mL}, 13 \mathrm{mmol}$ ) and trifluoroacetic anhydride (TFAA, 4 drops) under nitrogen atmosphere and the mixture was stirred at room temperature.

A solution of $14(0.50 \mathrm{mmol})$, TFAA (2 drops) in anhydrous dichloromethane $(100 \mathrm{~mL})$ was stirred at $-78^{\circ} \mathrm{C}$ under nitrogen atmosphere. To the above solution was added the prepared $\mathrm{VOF}_{3}$ solution $(4.9 \mathrm{~mL}, 1.3 \mathrm{mmol})$ over 10 minutes. After $1 \mathrm{~h}$, the reaction mixture was warmed to $-10{ }^{\circ} \mathrm{C}$ and further stirred for additional $1 \mathrm{~h}$ at the same temperature. After $1 \mathrm{~h}, 50 \mathrm{~mL}$ of $10 \% \mathrm{NaOH}$ solution were directly added to the reaction mixture and the reaction mixture was vigorously stirred at room temperature. After $1 \mathrm{~h}$, the reaction mixture was extracted with dichloromethane, and the organic layers were combined, dried over $\mathrm{MgSO}_{4}$, and concentrated. The crude mixture was purified by flash silica gel column chromatography using a 10:1 mixture of dichloromethane and methanol as the eluent to provide the natural product in group B.

\section{Deoxytylophorine (4)}<smiles>COc1ccc2c3c(c4cc(OC)c(OC)cc4c2c1)CN1CCCC1C3</smiles>

A yellow solid; $0.14 \mathrm{~g}, 78 \%$ yield. Spectroscopic data was in good match with a reported value in the literature. ${ }^{3}{ }^{1} \mathbf{H}$ NMR (500 MHz, $\left.\mathrm{CDCl}_{3}, \mathrm{ppm}\right): \delta 7.87-7.99(\mathrm{~m}, 3 \mathrm{H}), 7.22(\mathrm{~d}, J=9.2 \mathrm{~Hz}, 1 \mathrm{H})$, 
$7.17(\mathrm{~s}, 1 \mathrm{H}), 4.62(\mathrm{~d}, J=14.7 \mathrm{~Hz}, 1 \mathrm{H}), 4.11(\mathrm{~s}, 3 \mathrm{H}), 4.06(\mathrm{~s}, 3 \mathrm{H}), 4.02(\mathrm{~s}, 3 \mathrm{H}), 3.68(\mathrm{~d}, J=14.7 \mathrm{~Hz}$, 1H), $3.49(\mathrm{t}, J=8.2 \mathrm{~Hz}, 1 \mathrm{H}), 3.4(\mathrm{~d}, J=15.0 \mathrm{~Hz}, 1 \mathrm{H}), 2.91-3.00(\mathrm{~m}, 1 \mathrm{H}), 2.44-2.55(\mathrm{~m}, 2 \mathrm{H}), 2.20$ - $2.28(\mathrm{~m}, 1 \mathrm{H}), 2.00-2.08(\mathrm{~m}, 1 \mathrm{H}), 1.88-1.97(\mathrm{~m}, 1 \mathrm{H}), 1.72-1.82(\mathrm{~m}, 1 \mathrm{H}) ;{ }^{13} \mathrm{C}$ NMR $(125 \mathrm{MHz}$, $\left.\mathrm{CDCl}_{3}, \mathrm{ppm}\right): \delta 157.7,149.6,148.4,130.5,127.2,125.8,125.7,125.4,125.3,123.5,114.9,104.8$, 104.1, 103.3, 60.4, 56.2, 56.1, 55.7, 55.3, 54.1, 33.7, 31.4, 21.8; IR (film): 2924, 2852, 1616, 1512, 1468, 1414, 1260, 1204, 1166, 1032, 840, $750 \mathrm{~cm}^{-1}$; HRMS (ESI) calcd for $\mathrm{C}_{23} \mathrm{H}_{25} \mathrm{NO}_{3} \mathrm{Na}[\mathrm{M}+\mathrm{Na}]$ 386.1732 , found 386.1737 .

\section{Isotylocrebrine (5)}

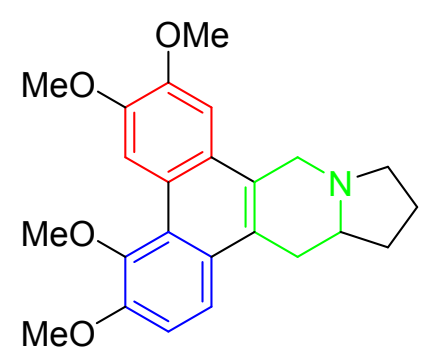

A yellow solid; $0.11 \mathrm{~g}, 58 \%$ yield. Spectroscopic data was in good match with a reported value in the literature. ${ }^{3}{ }^{1} \mathbf{H}$ NMR $\left(500 \mathrm{MHz}, \mathrm{CDCl}_{3}, \mathrm{ppm}\right): \delta 9.33(\mathrm{~s}, 1 \mathrm{H}), 7.66(\mathrm{~d}, J=9.2 \mathrm{~Hz}, 1 \mathrm{H}), 7.33(\mathrm{~s}, 1 \mathrm{H})$, $7.29(\mathrm{~d}, J=9.2 \mathrm{~Hz}, 1 \mathrm{H}), 4.67(\mathrm{~d}, J=15.0 \mathrm{~Hz}, 1 \mathrm{H}), 4.07(\mathrm{~s}, 6 \mathrm{H}), 4.04$ (s, 3H), 3.92 (s, 3H), 3.69 (d, $\mathrm{J}=14.7 \mathrm{~Hz}, 1 \mathrm{H}), 3.44-3.50(\mathrm{~m}, 1 \mathrm{H}), 3.34(\mathrm{dd}, J=15.7,2.3 \mathrm{~Hz}, 1 \mathrm{H}), 2.88-2.97(\mathrm{~m}, 1 \mathrm{H}), 2.43-2.54$ $(\mathrm{m}, 2 \mathrm{H}), 2.21-2.29(\mathrm{~m}, 1 \mathrm{H}), 2.00-2.08(\mathrm{~m}, 1 \mathrm{H}), 1.88-1.98(\mathrm{~m}, 1 \mathrm{H}), 1.75-1.84(\mathrm{~m}, 1 \mathrm{H}) ;{ }^{13} \mathrm{C}$ NMR (125 MHz, $\left.\mathrm{CDCl}_{3}, \mathrm{ppm}\right): \delta$ 150.7, 148.8, 147.9, 146.4, 126.6, 125.9, 123.7, 123.4, 119.0, 112.2, 109.3, 103.6, 60.4, 60.2, 56.6, 55.9, 55.3, 54.4, 34.1, 31.4, 21.8; IR (film): 2918, 2846, 1515, 1466, 1260, 1064, $790 \mathrm{~cm}^{-1}$; HRMS (ESI) calcd for $\mathrm{C}_{24} \mathrm{H}_{27} \mathrm{NO}_{4} \mathrm{Na}[\mathrm{M}+\mathrm{Na}]$ 416.1838, found 416.1839. 


\section{References}

1. Lee, C.-Y.; Cheon, C.-H. Adv. Synth. Catal. 2017, 359, 3831.

2. Zhang, B.; Chen, R.; Jiang, H.; Zhou, Q.; Qiu, F.; Han, D.; Li, R.; Tang, W.; Zhong, A.; Zhang, J.; Yu, X. Tetrahedron 2016, 72, 2813.

3. Niphakis, M. J.; Georg, G. I. Org. Lett. 2011, 13, 196. 


\section{Spectroscopic Data}

5-1. NMR Spectrum of ortho-Bromoaryl MIDA Boronate 9

a) ${ }^{1} \mathrm{H}$ NMR Spectrum (in DMSO-d 6 , $500 \mathrm{MHz}$ )

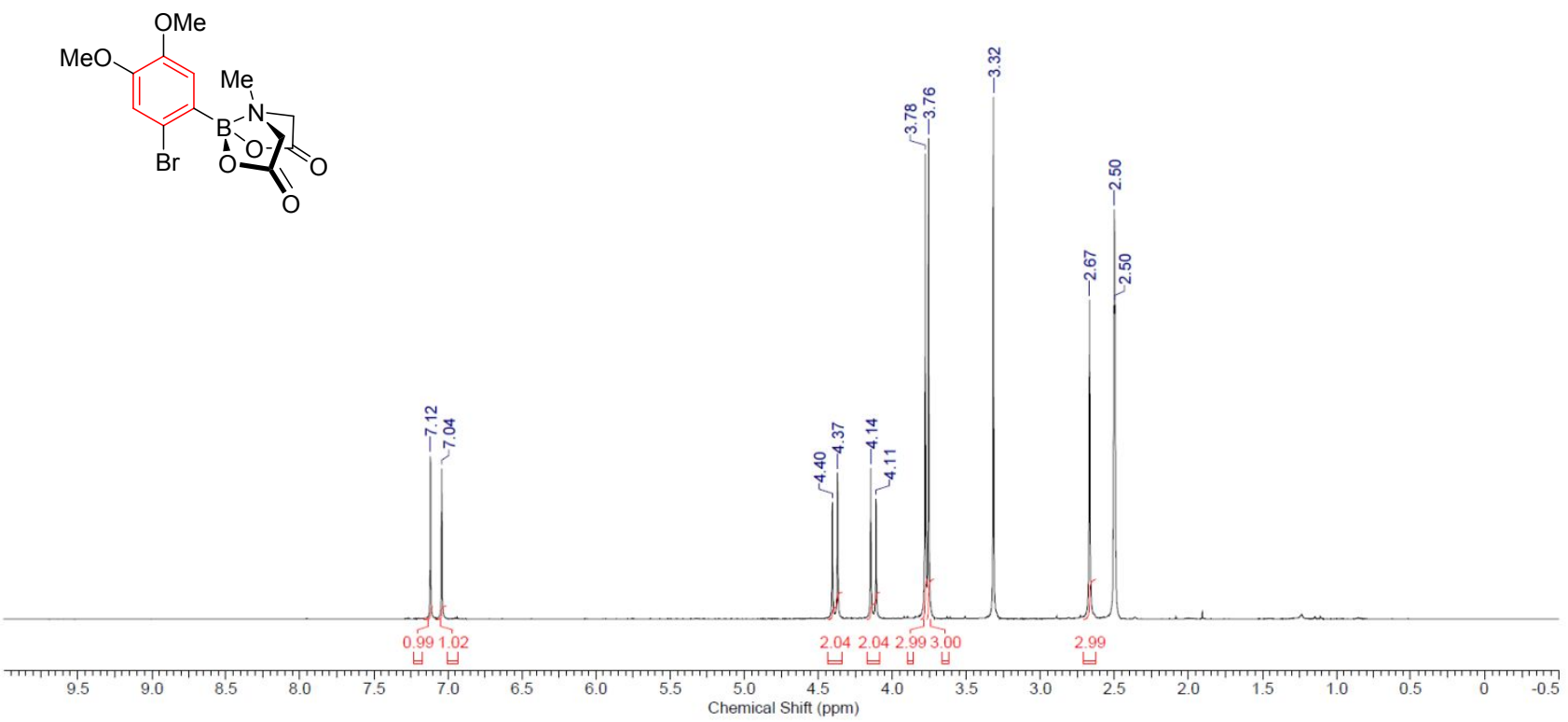


5-2. NMR Spectra of 3-(4-Bromopyridin-2-yl)prop-2-yn-1-ol (10a)

a) ${ }^{1} \mathrm{H}$ NMR Spectrum (in $\mathrm{CDCl}_{3}, 500 \mathrm{MHz}$ )
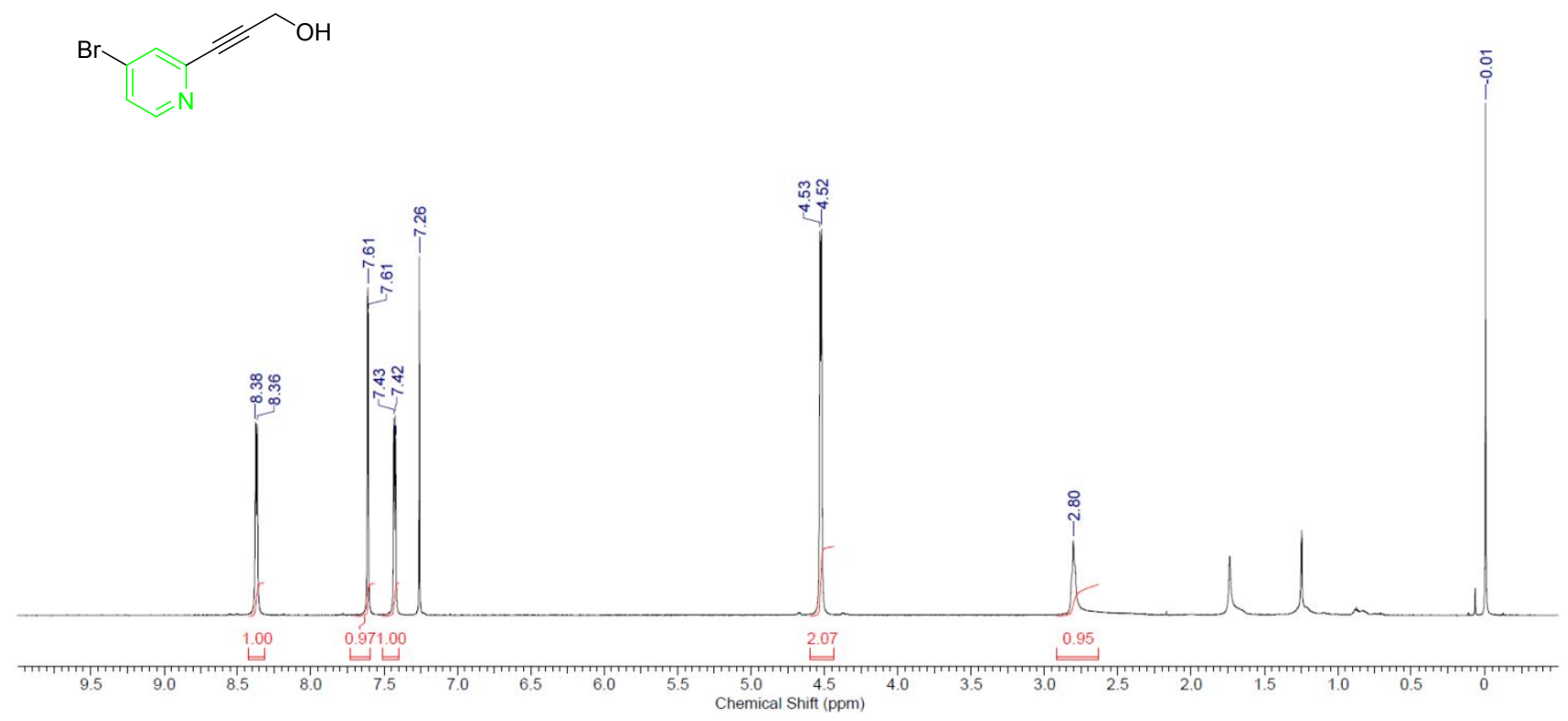

b) ${ }^{13} \mathrm{C}$ NMR Spectrum (in $\mathrm{CDCl}_{3}, 125 \mathrm{MHz}$ )

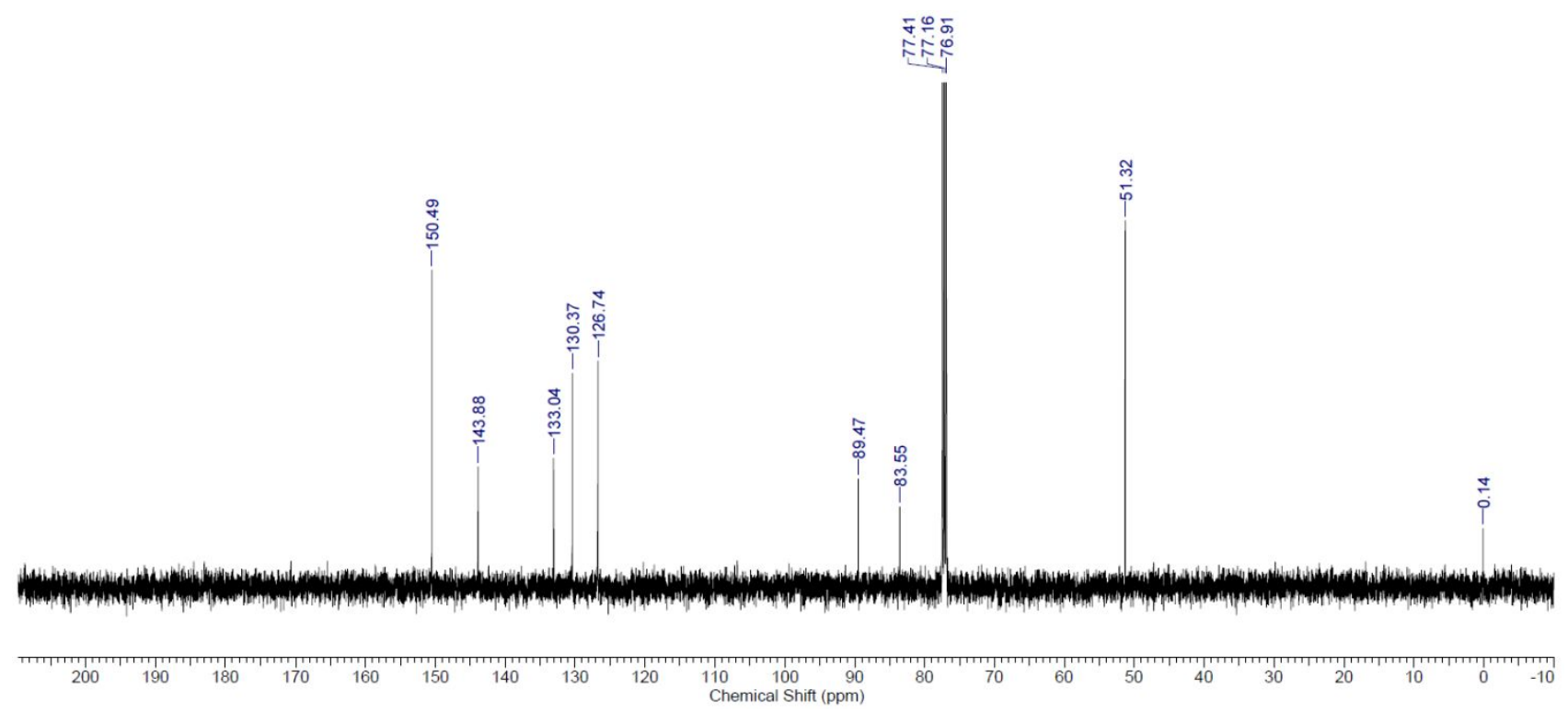


5-3. NMR Spectrum of 3-(Pyridin-2-yl)propan-1-ol (red-10a)

a) ${ }^{1} \mathrm{H}$ NMR Spectrum (in $\mathrm{CDCl}_{3}, 500 \mathrm{MHz}$ )

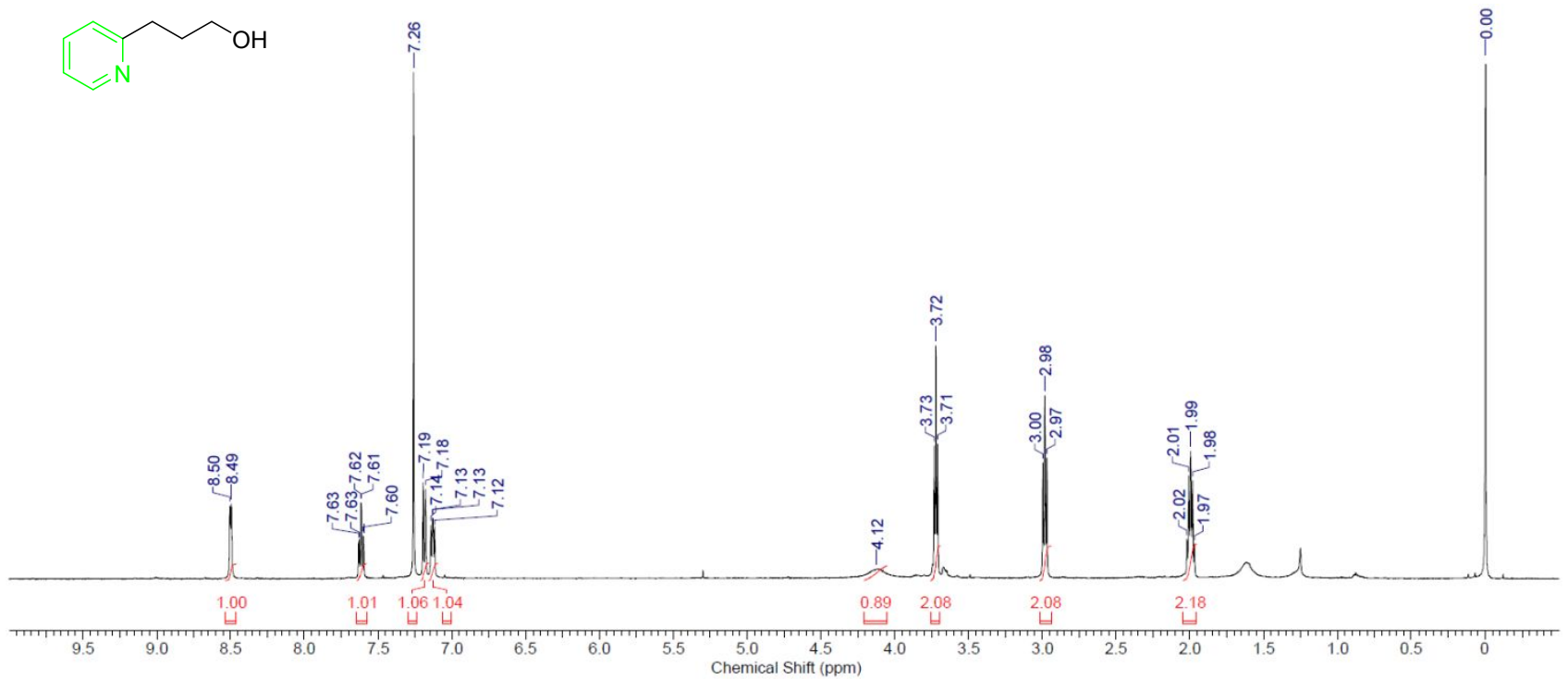


5-4. NMR Spectrum of 3-(5-Bromopyridin-2-yl)prop-2-yn-1-ol (10b)

a) ${ }^{1} \mathrm{H}$ NMR Spectrum (in $\mathrm{CDCl}_{3}, 500 \mathrm{MHz}$ )
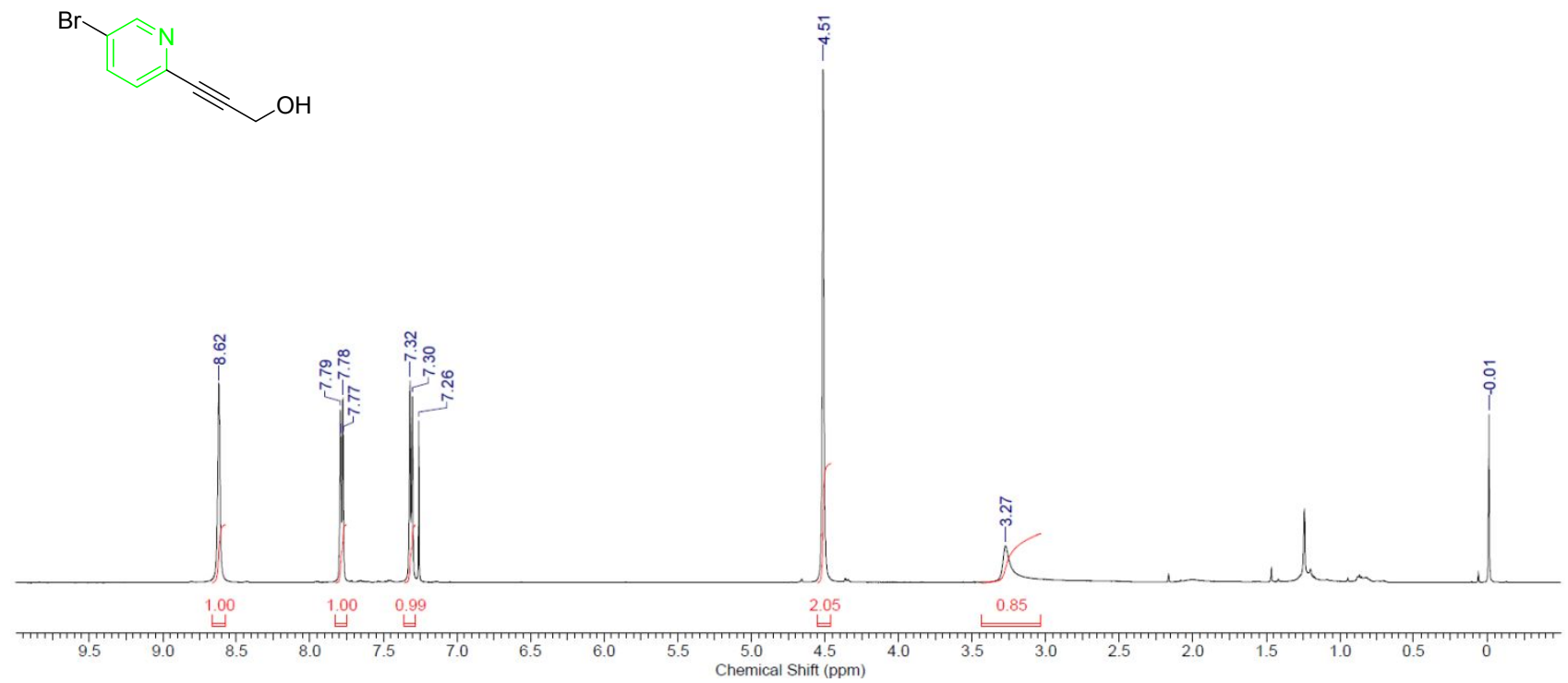
5-5. NMR Spectra of Compound 13a

a) ${ }^{1} \mathrm{H}$ NMR Spectrum (in $\mathrm{CDCl}_{3}, 500 \mathrm{MHz}$ )

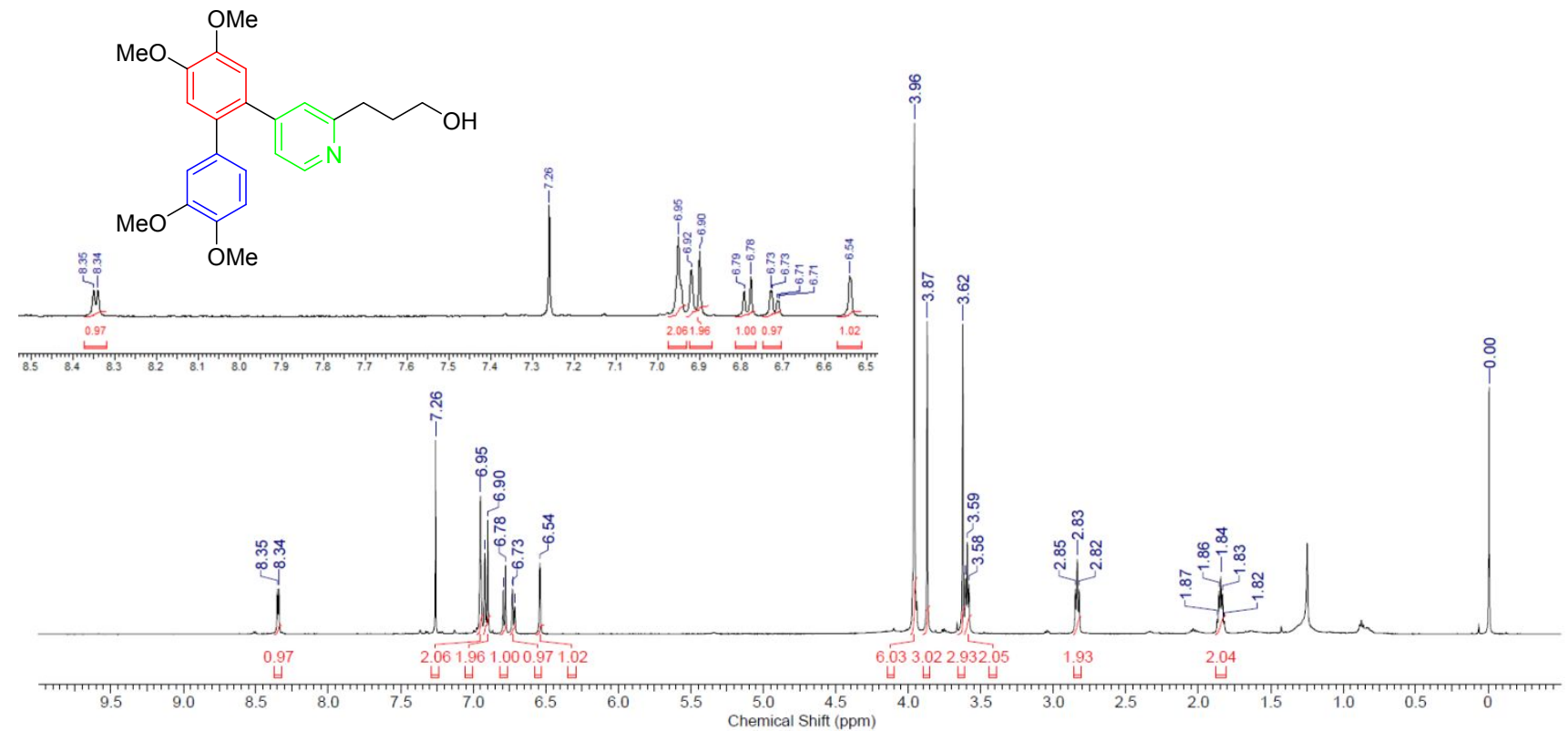

b) ${ }^{13} \mathrm{C}$ NMR Spectrum (in $\mathrm{CDCl}_{3}, 125 \mathrm{MHz}$ )

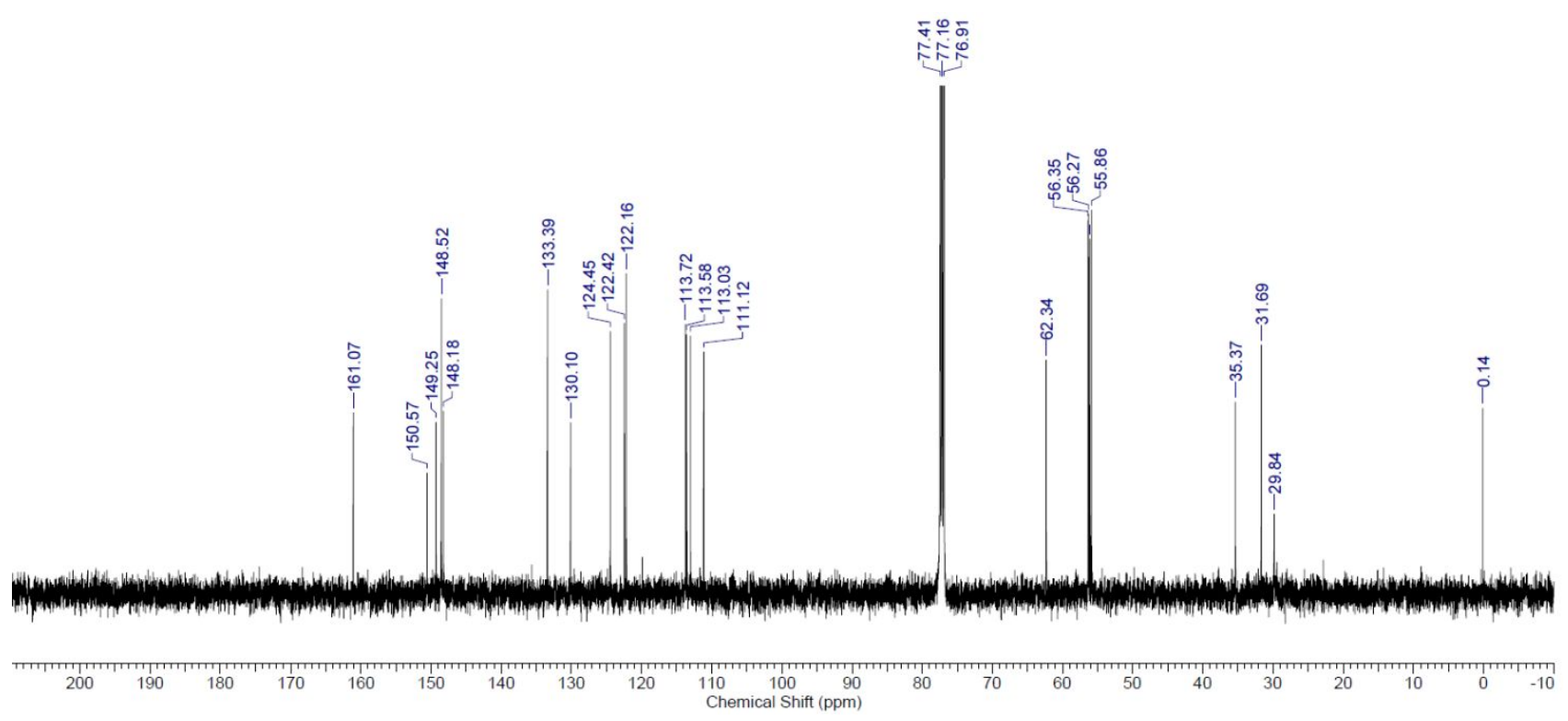


5-6. NMR Spectra of Compound 13b

a) ${ }^{1} \mathrm{H}$ NMR Spectrum (in $\mathrm{CDCl}_{3}, 500 \mathrm{MHz}$ )

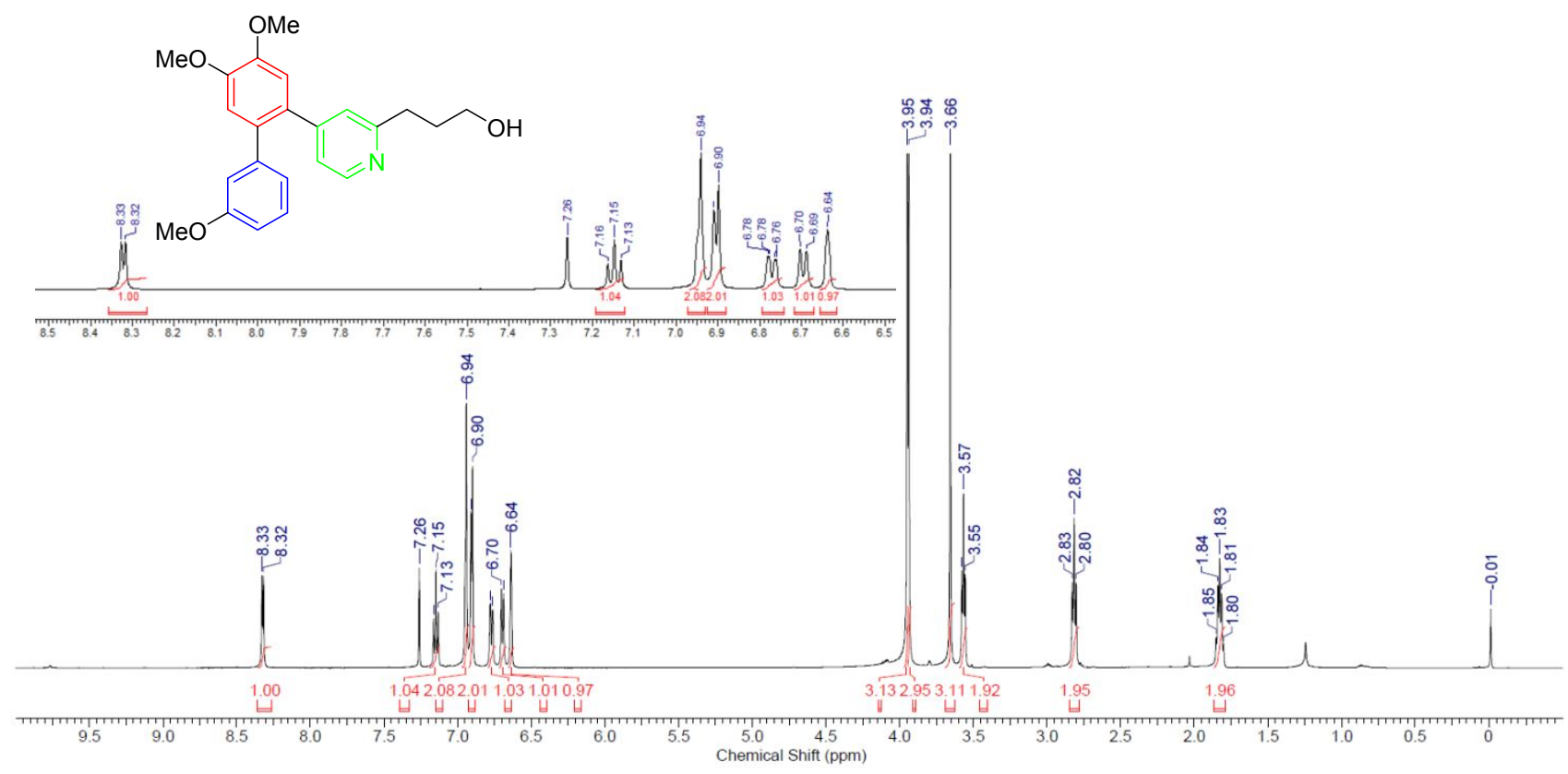

b) ${ }^{13} \mathrm{C}$ NMR Spectrum (in $\mathrm{CDCl}_{3}, 125 \mathrm{MHz}$ )

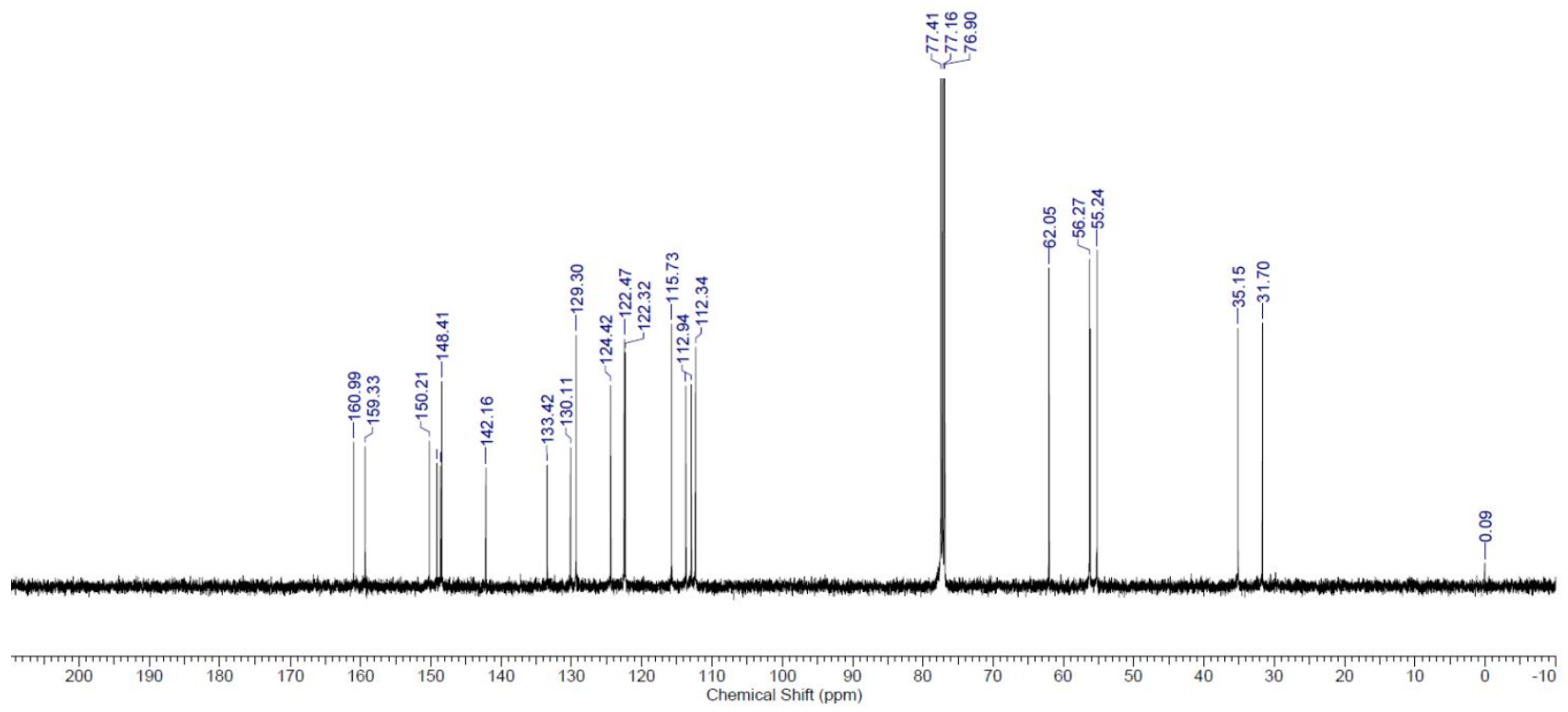


5-7. NMR Spectra of Compound 13c

a) ${ }^{1} \mathrm{H}$ NMR Spectrum (in $\mathrm{CDCl}_{3}, 500 \mathrm{MHz}$ )

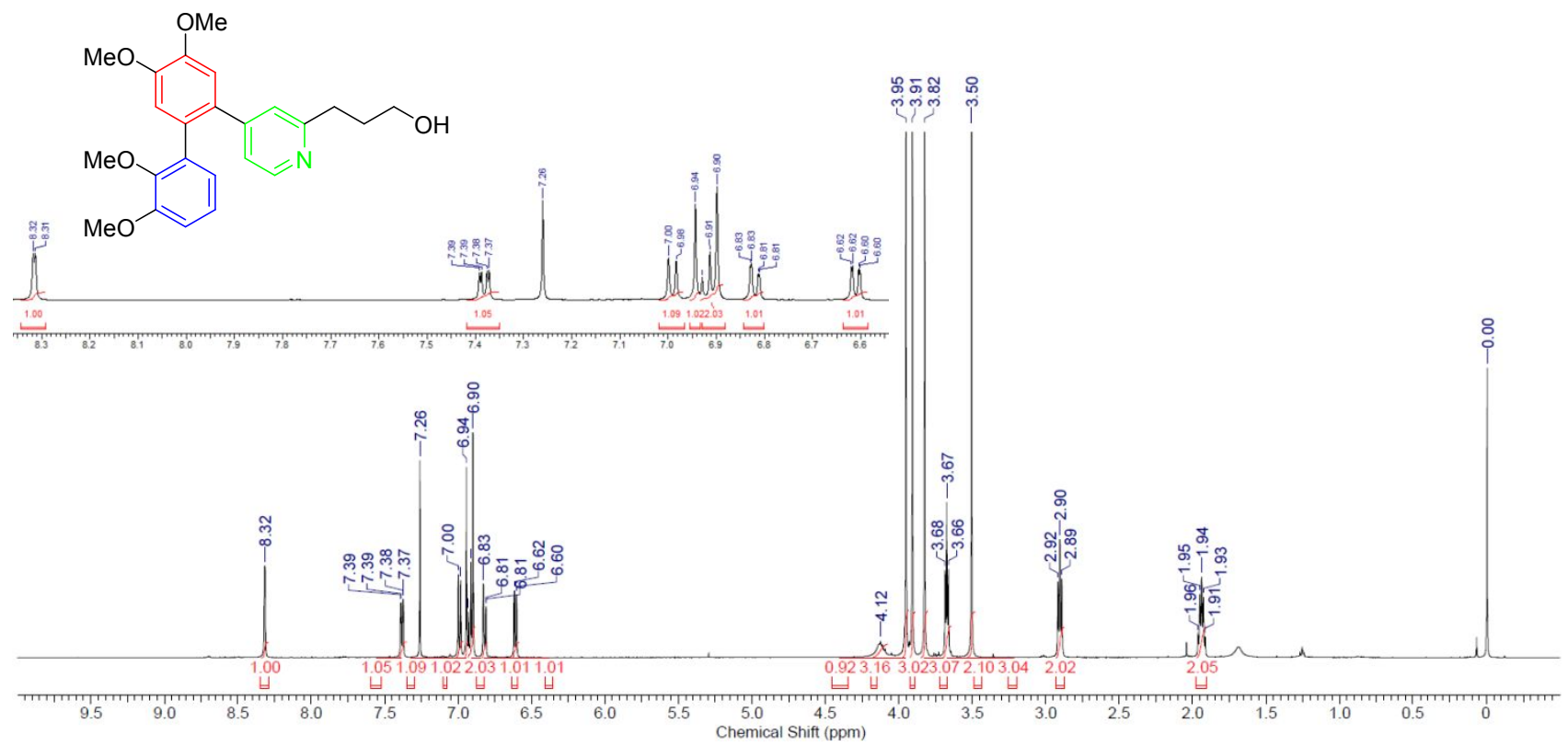

b) ${ }^{13} \mathrm{C}$ NMR Spectrum (in $\mathrm{CDCl}_{3}, 125 \mathrm{MHz}$ )
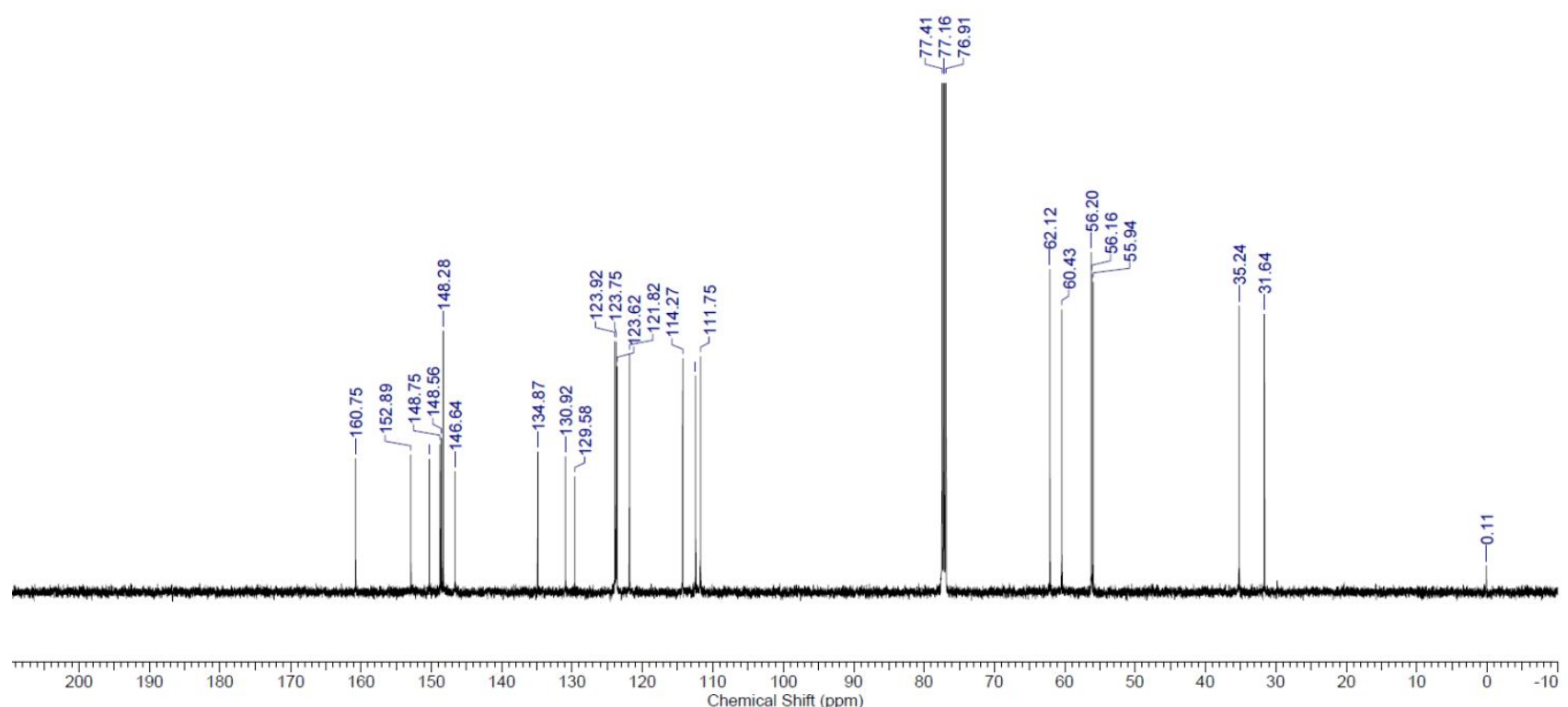
5-8. NMR Spectrum of Compound 14a

a) ${ }^{1} \mathrm{H}$ NMR Spectrum (in $\mathrm{CDCl}_{3}, 500 \mathrm{MHz}$ )
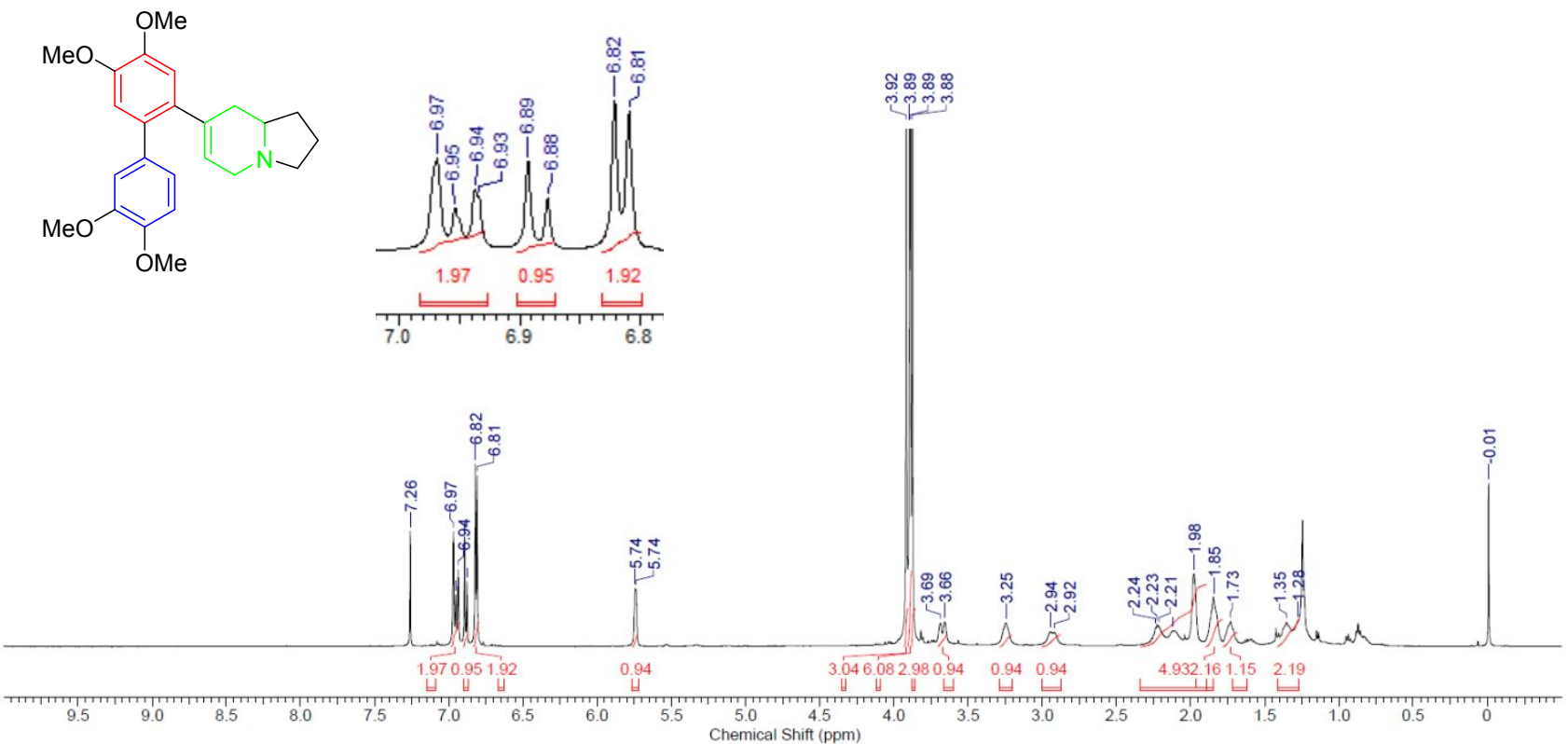
5-9. NMR Spectrum of Compound 14b

a) ${ }^{1} \mathrm{H}$ NMR Spectrum (in $\mathrm{CDCl}_{3}, 500 \mathrm{MHz}$ )

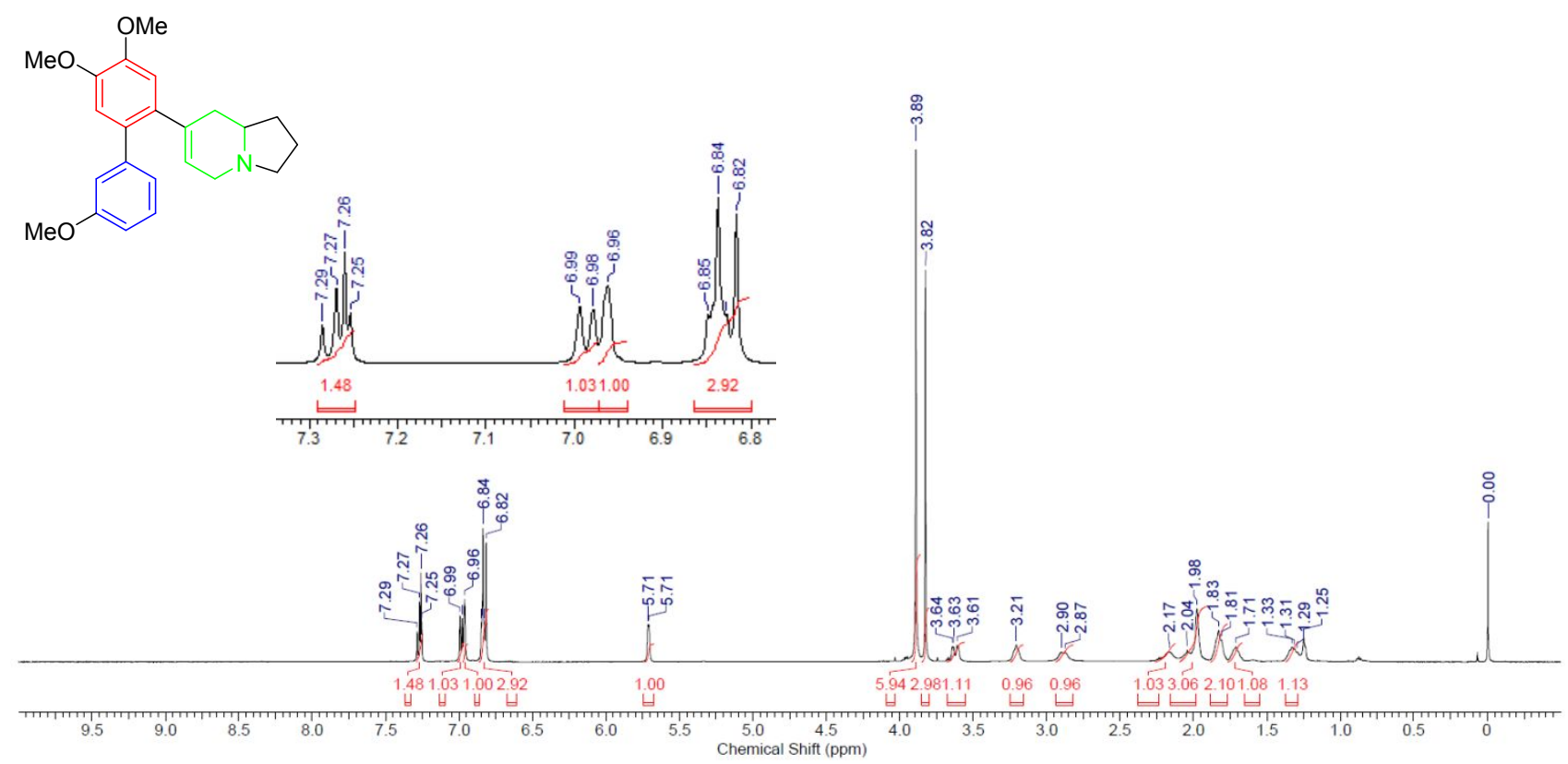


5-10. NMR Spectrum of Compound 14c

a) ${ }^{1} \mathrm{H}$ NMR Spectrum (in $\mathrm{CDCl}_{3}, 500 \mathrm{MHz}$ )

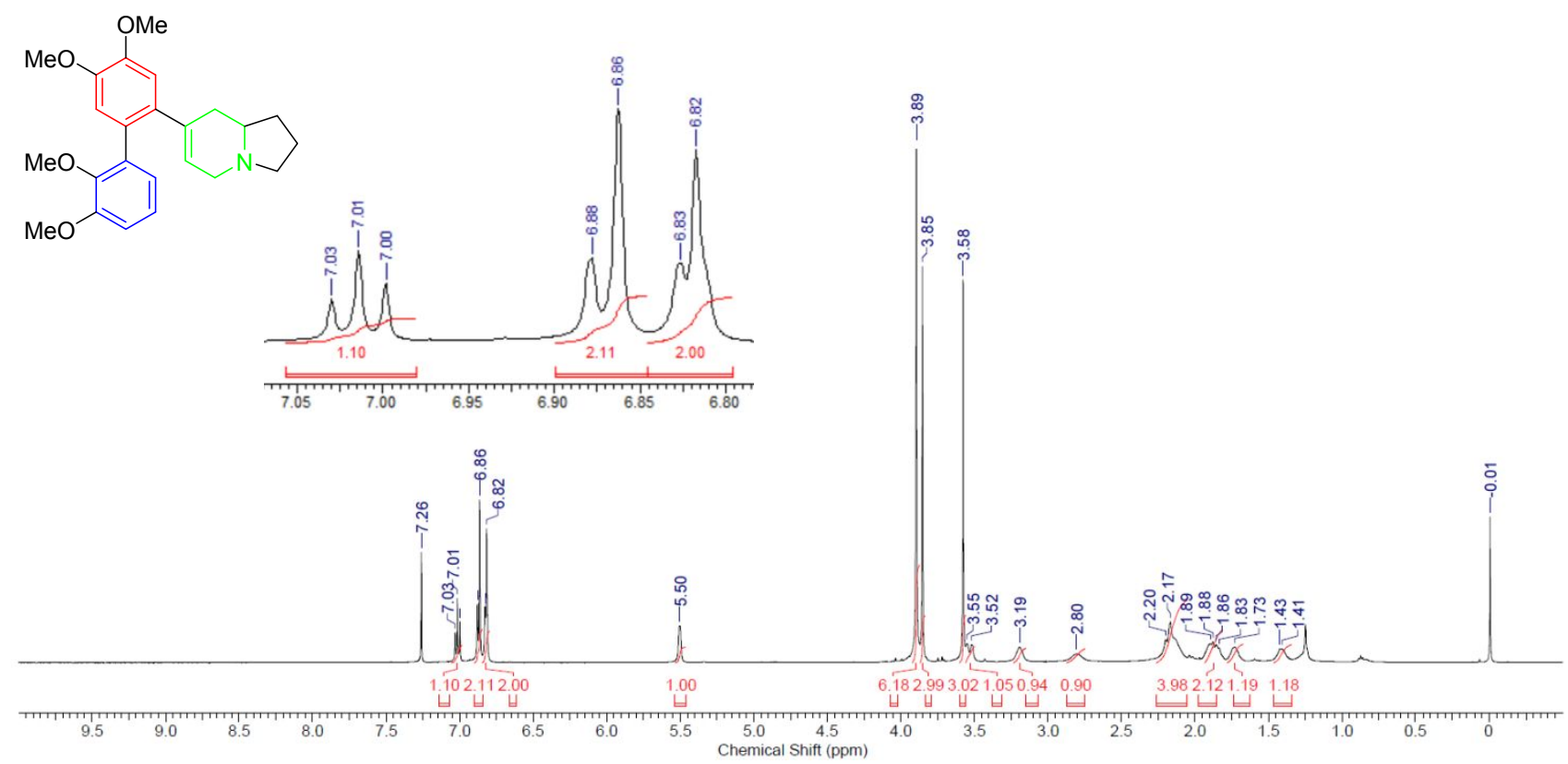


5-11. NMR Spectra of Tylophorine (1)

a) ${ }^{1} \mathrm{H}$ NMR Spectrum (in $\mathrm{CDCl}_{3}, 500 \mathrm{MHz}$ )

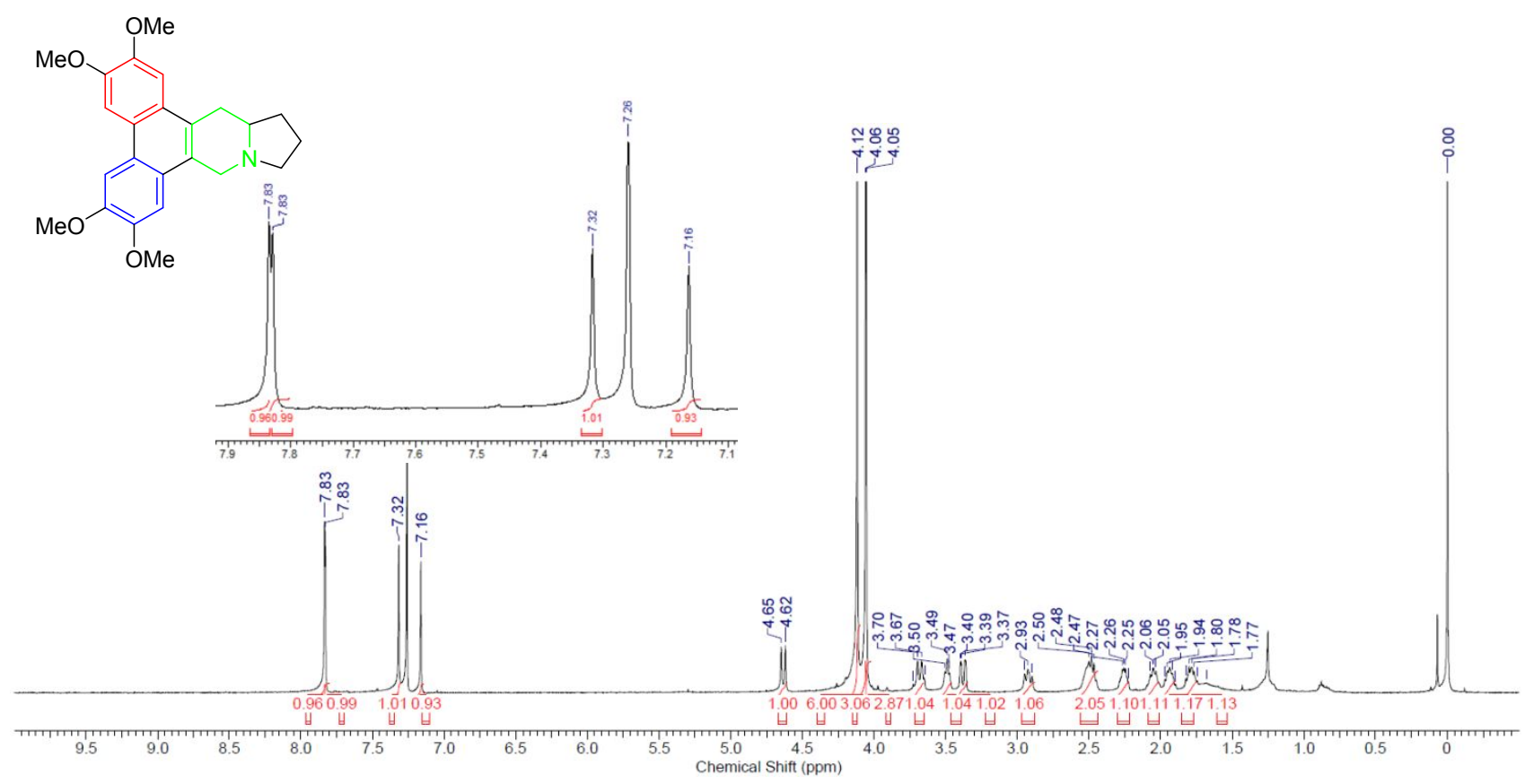

b) ${ }^{13} \mathrm{C}$ NMR Spectrum (in $\mathrm{CDCl}_{3}, 125 \mathrm{MHz}$ )
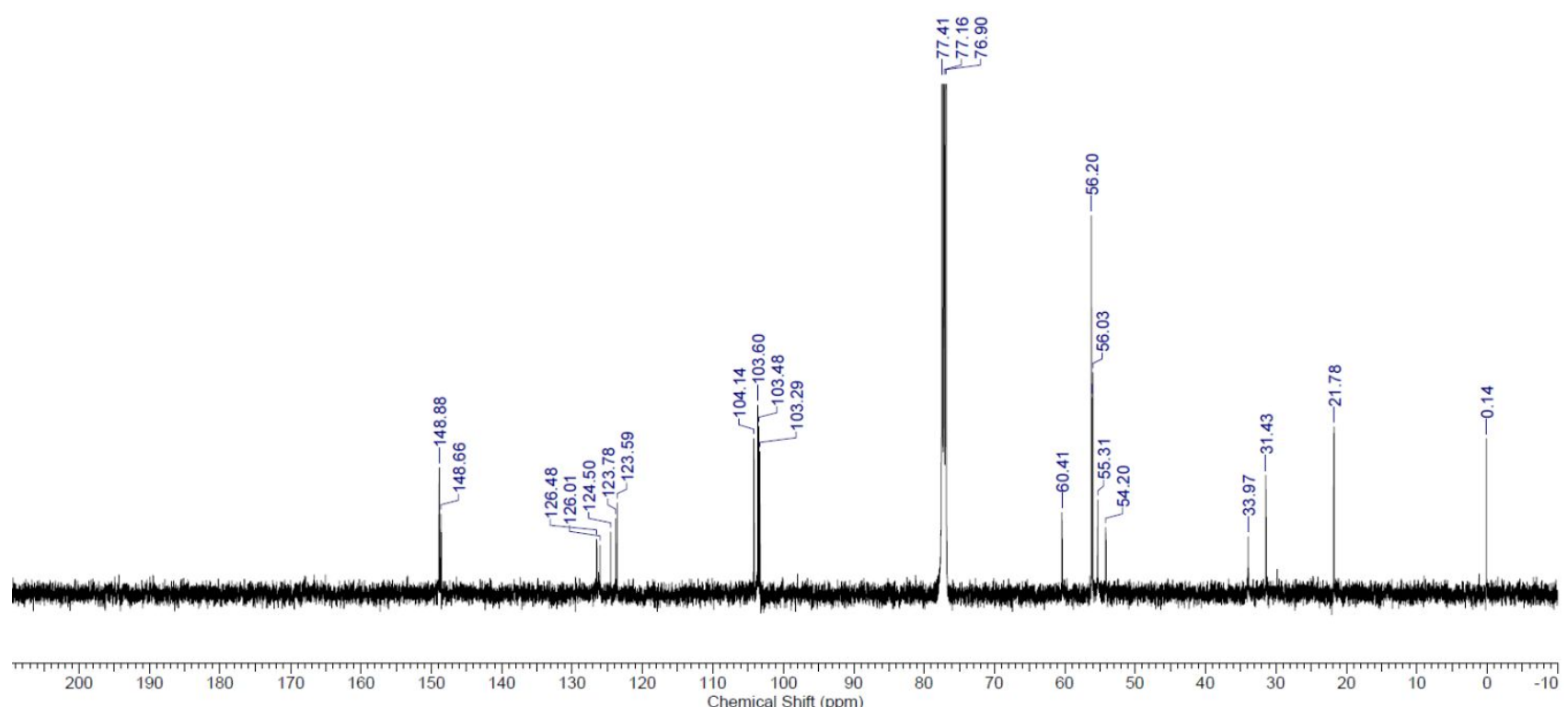
5-12. NMR Spectra of Antofine (2)

a) ${ }^{1} \mathrm{H}$ NMR Spectrum (in $\mathrm{CDCl}_{3}, 500 \mathrm{MHz}$ )

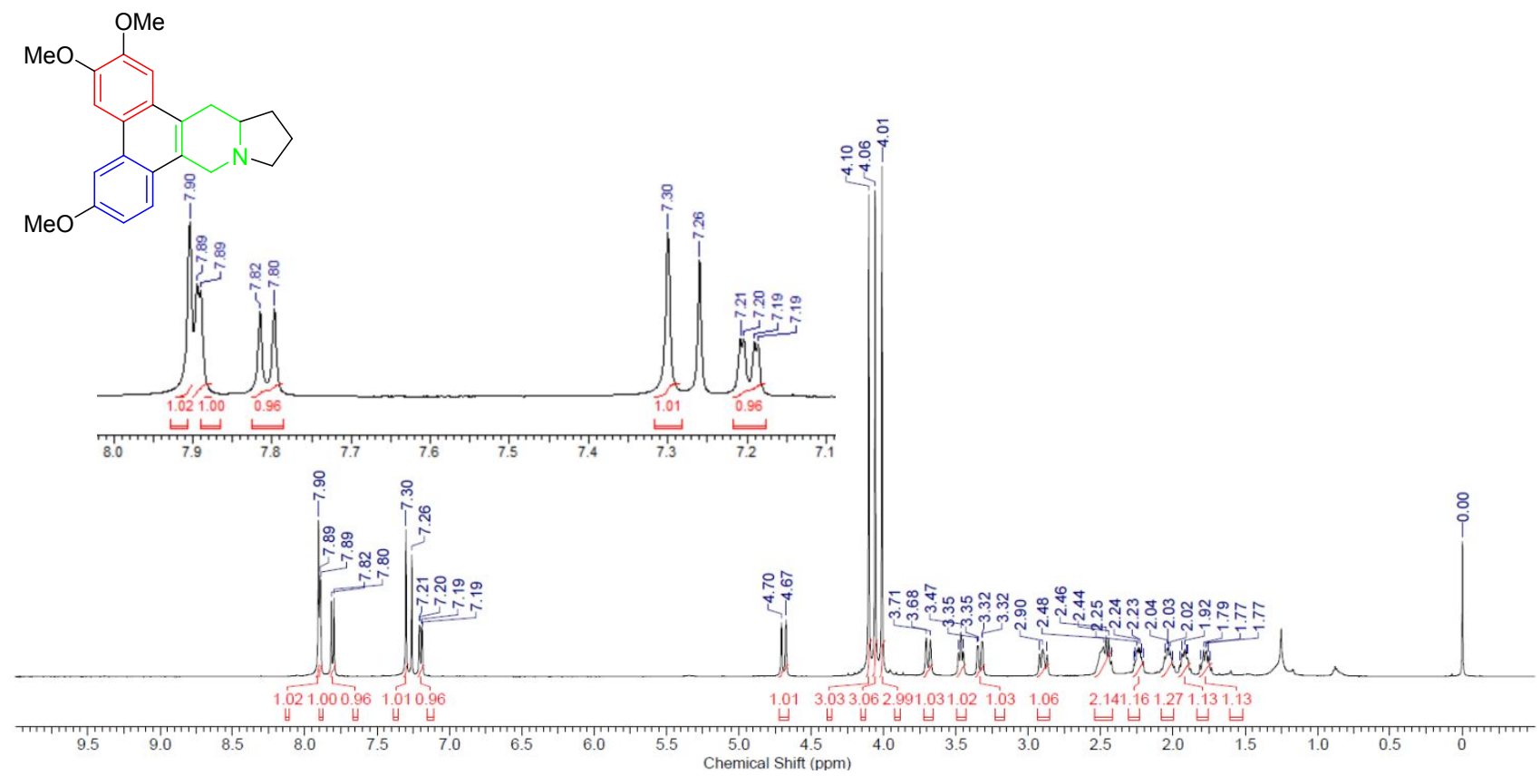

b) ${ }^{13} \mathrm{C}$ NMR Spectrum (in $\mathrm{CDCl}_{3}, 125 \mathrm{MHz}$ )
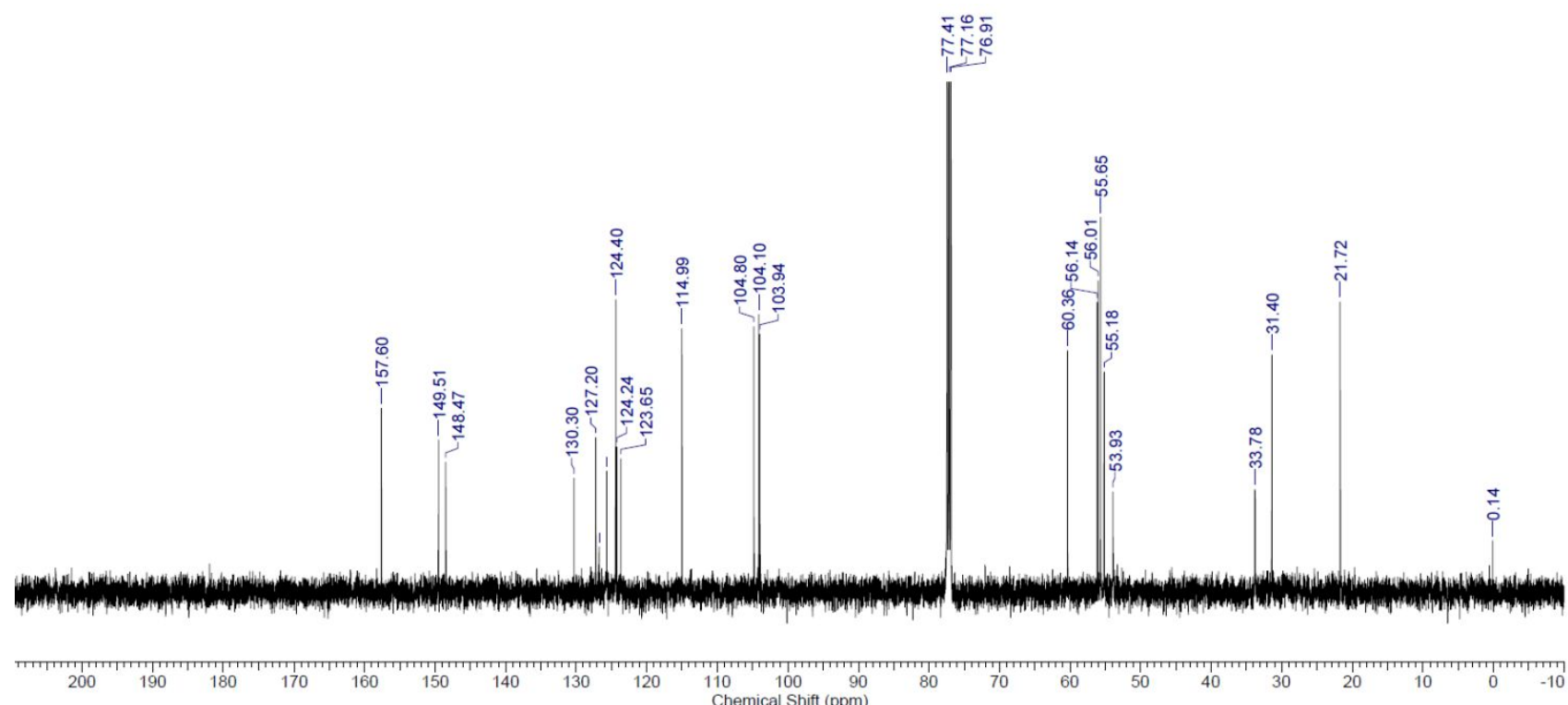
5-13. NMR Spectra of Tylocrebrine (3)

a) ${ }^{1} \mathrm{H}$ NMR Spectrum (in $\mathrm{CDCl}_{3}, 500 \mathrm{MHz}$ )<smiles></smiles><smiles>CCCCCCC</smiles>
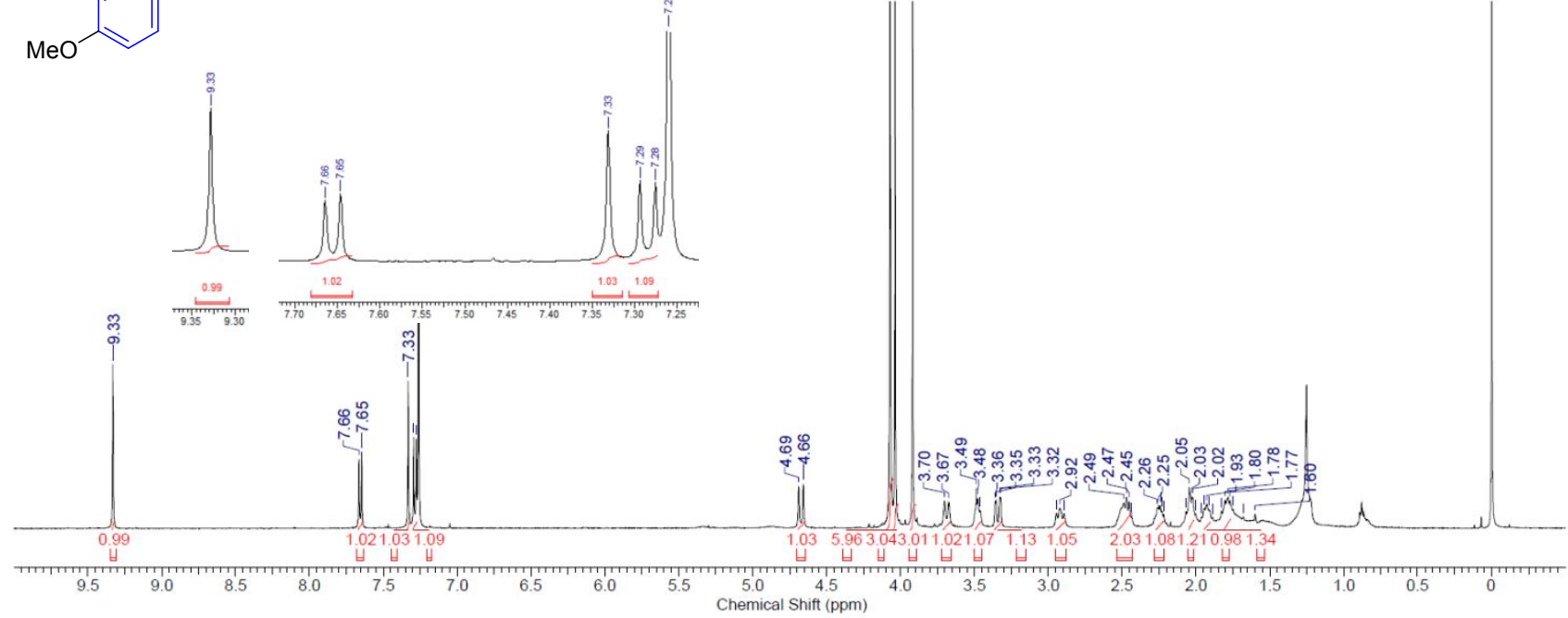

b) ${ }^{13} \mathrm{C}$ NMR Spectrum (in $\mathrm{CDCl}_{3}, 125 \mathrm{MHz}$ )

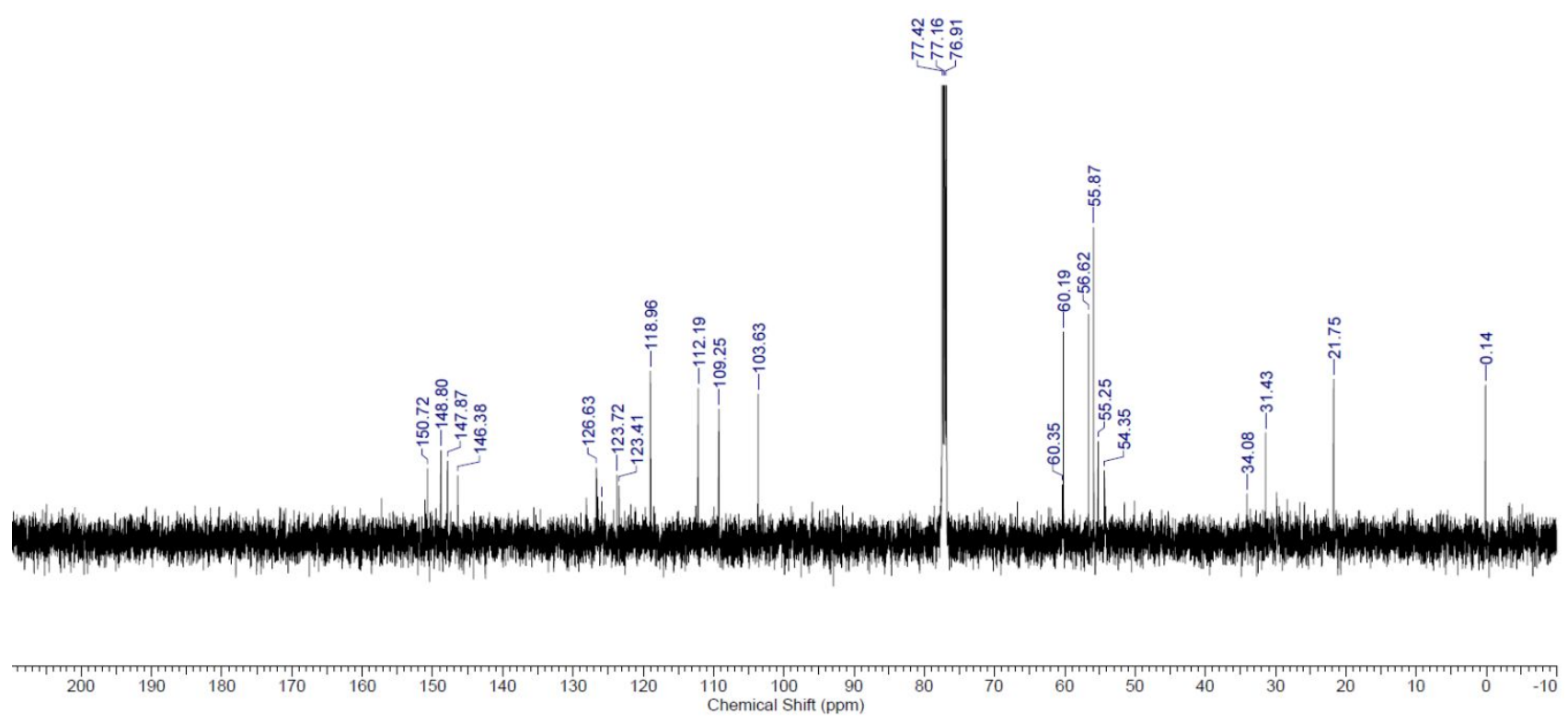


5-14. NMR Spectra of Compound 13d

a) ${ }^{1} \mathrm{H}$ NMR Spectrum (in $\mathrm{CDCl}_{3}, 500 \mathrm{MHz}$ )

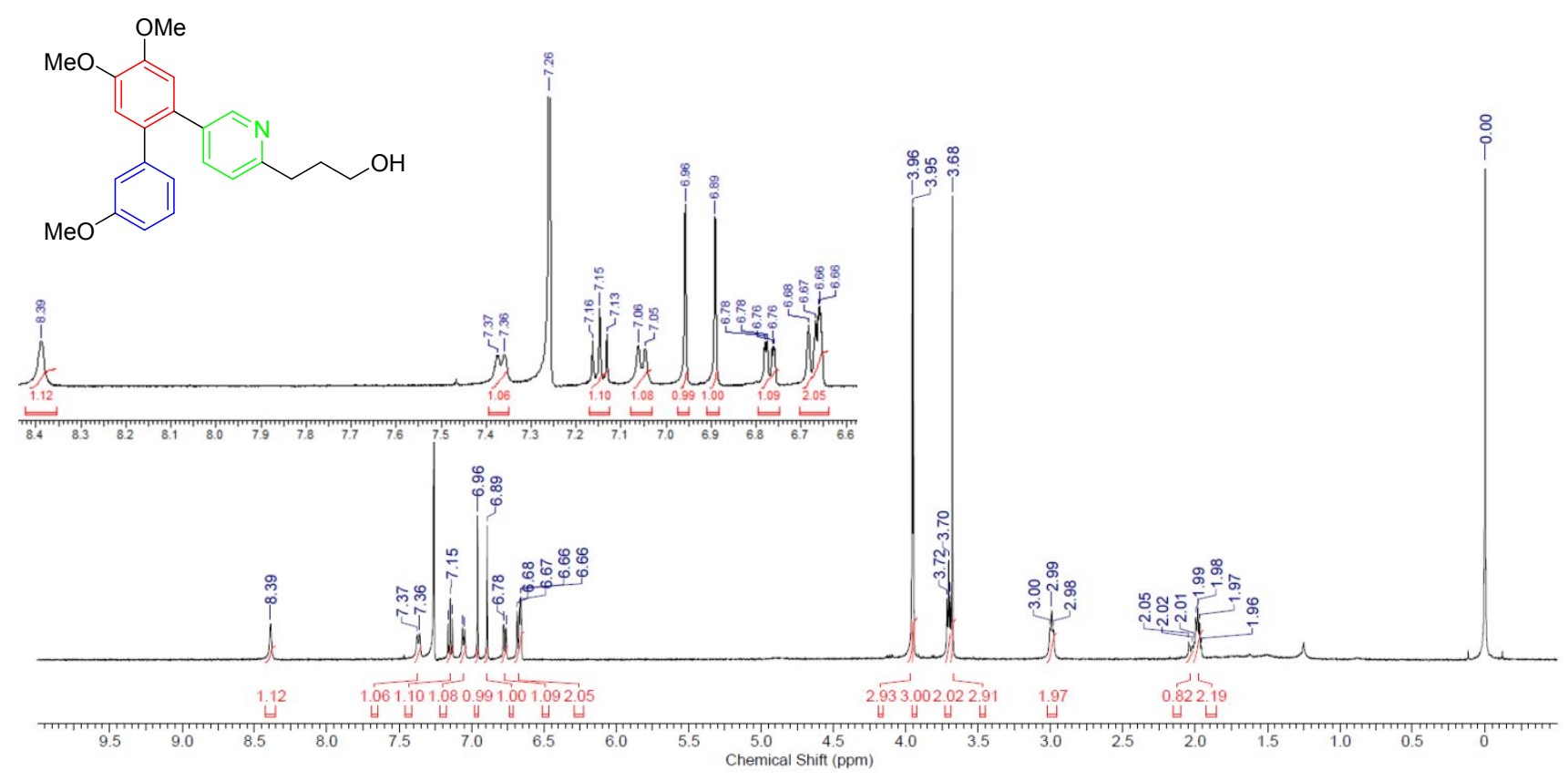

b) ${ }^{13} \mathrm{C}$ NMR Spectrum (in $\mathrm{CDCl}_{3}, 125 \mathrm{MHz}$ )

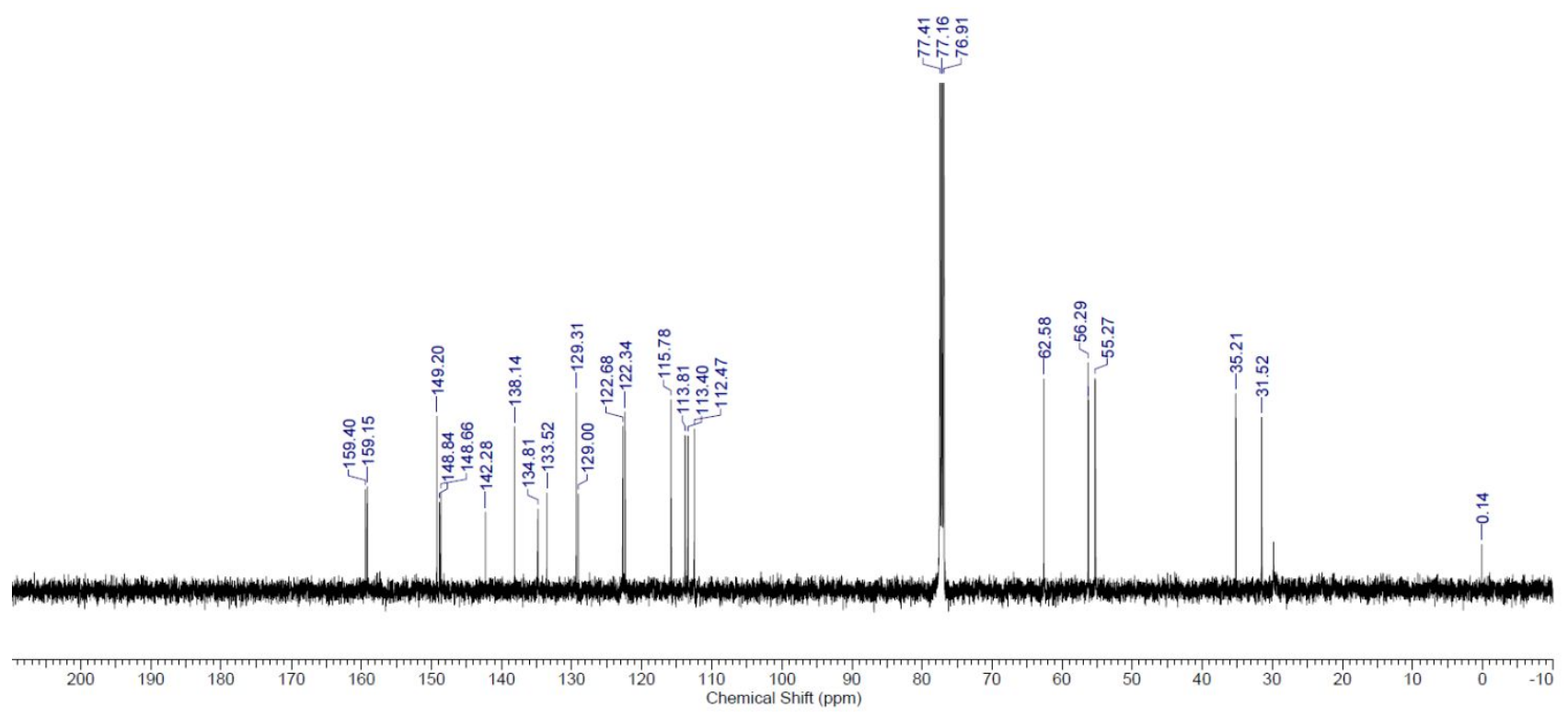


5-15. NMR Spectra of Compound 13e

a) ${ }^{1} \mathrm{H}$ NMR Spectrum (in $\mathrm{CDCl}_{3}, 500 \mathrm{MHz}$ )

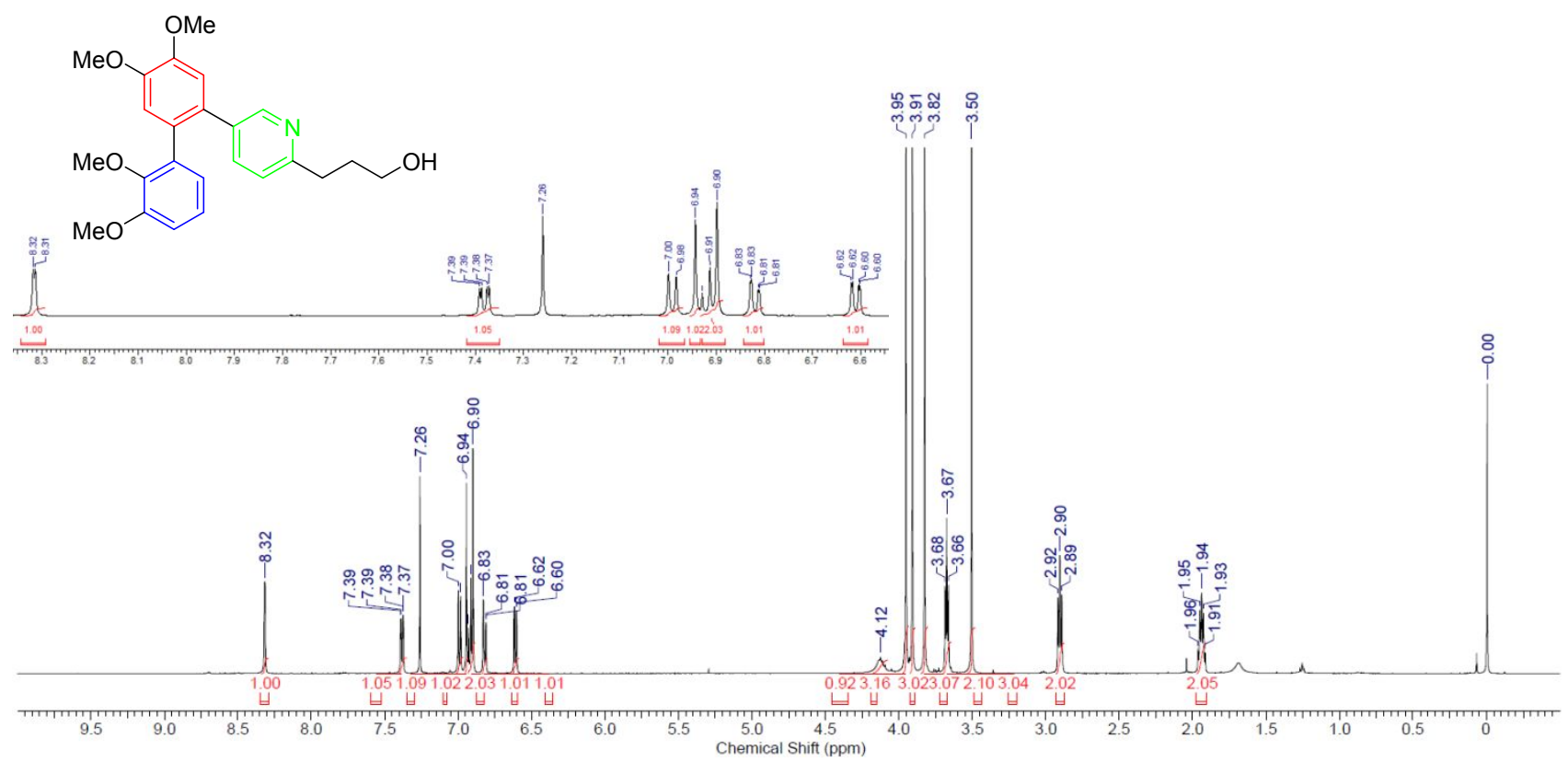

b) ${ }^{13} \mathrm{C}$ NMR Spectrum (in $\mathrm{CDCl}_{3}, 125 \mathrm{MHz}$ )
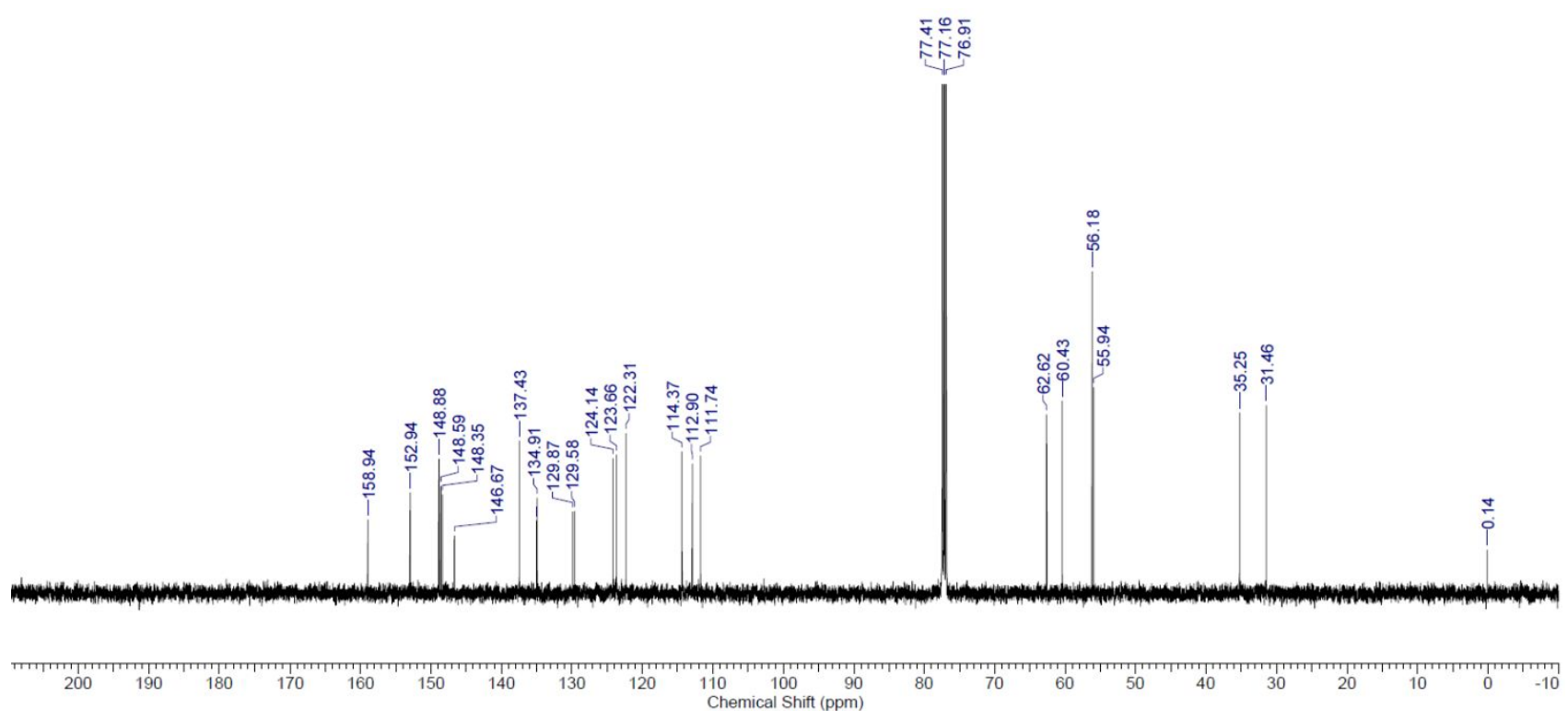
5-16. NMR Spectra of Compound 14d

a) ${ }^{1} \mathrm{H}$ NMR Spectrum (in $\mathrm{CDCl}_{3}, 500 \mathrm{MHz}$ )

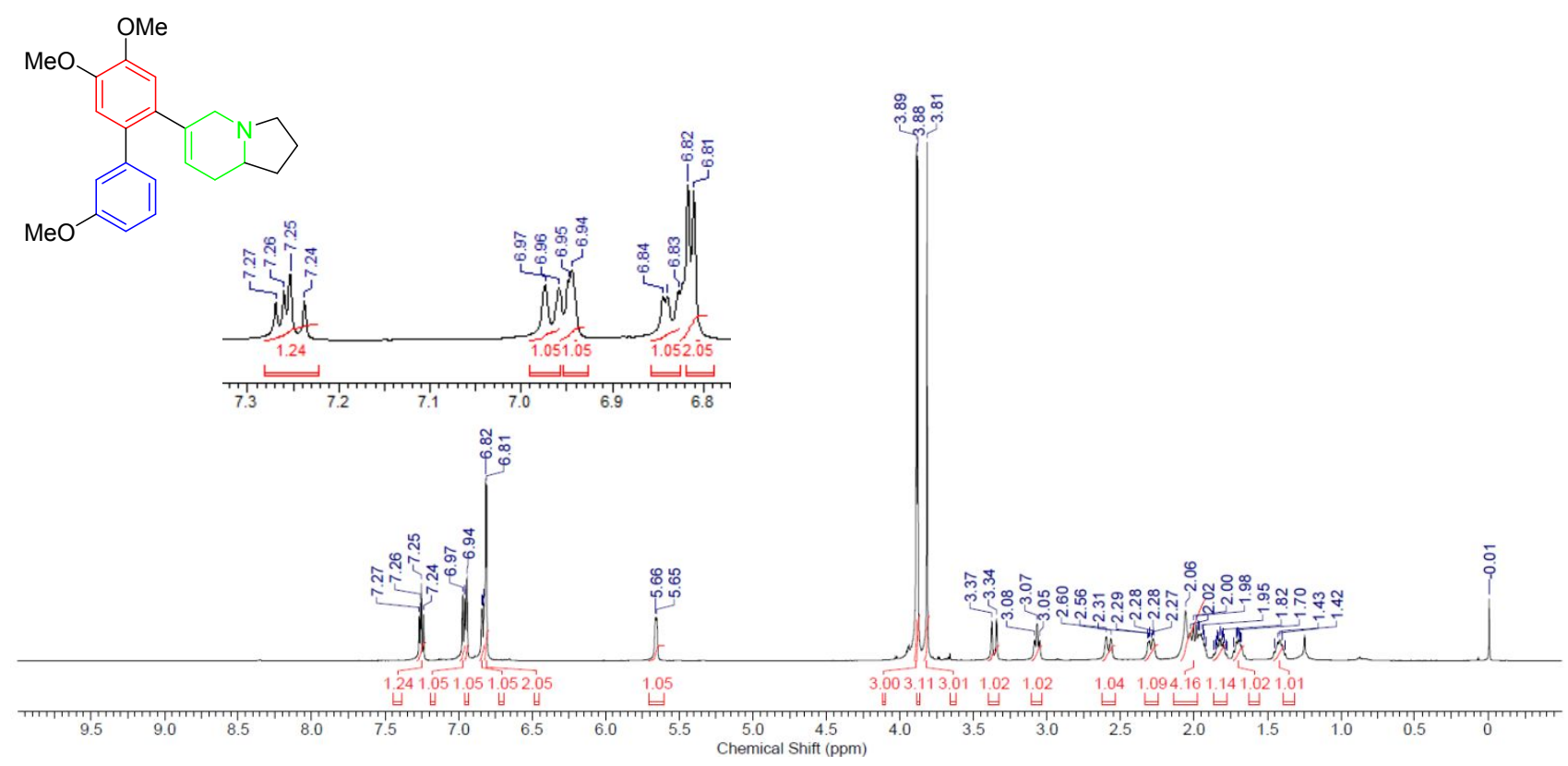

b) ${ }^{13} \mathrm{C}$ NMR Spectrum (in $\mathrm{CDCl}_{3}, 125 \mathrm{MHz}$ )

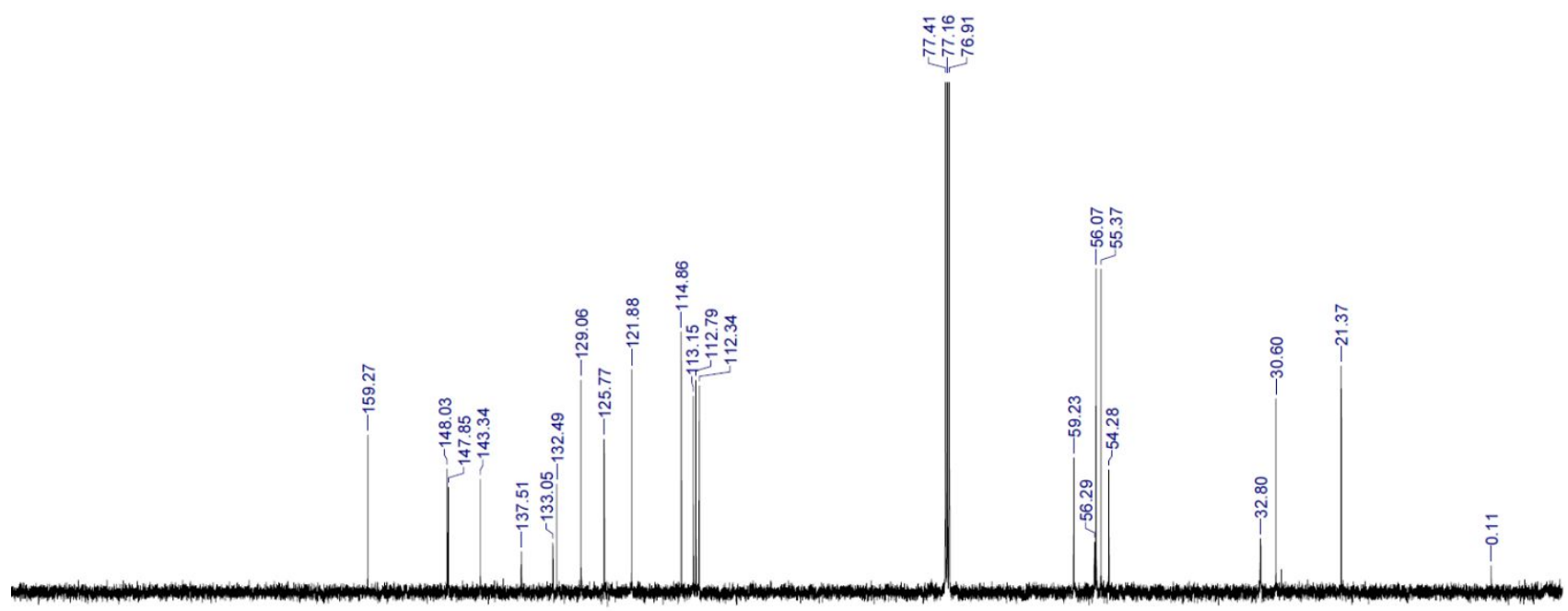

$\begin{array}{llllllllll}200 & 190 & 180 & 170 & 160 & 150 & 140 & 130 & 120 & 110 \\ \text { Chemical Shift (ppm) }\end{array}$ 
5-17. NMR Spectra of Compound 14e

a) ${ }^{1} \mathrm{H}$ NMR Spectrum (in $\mathrm{CDCl}_{3}, 500 \mathrm{MHz}$ )

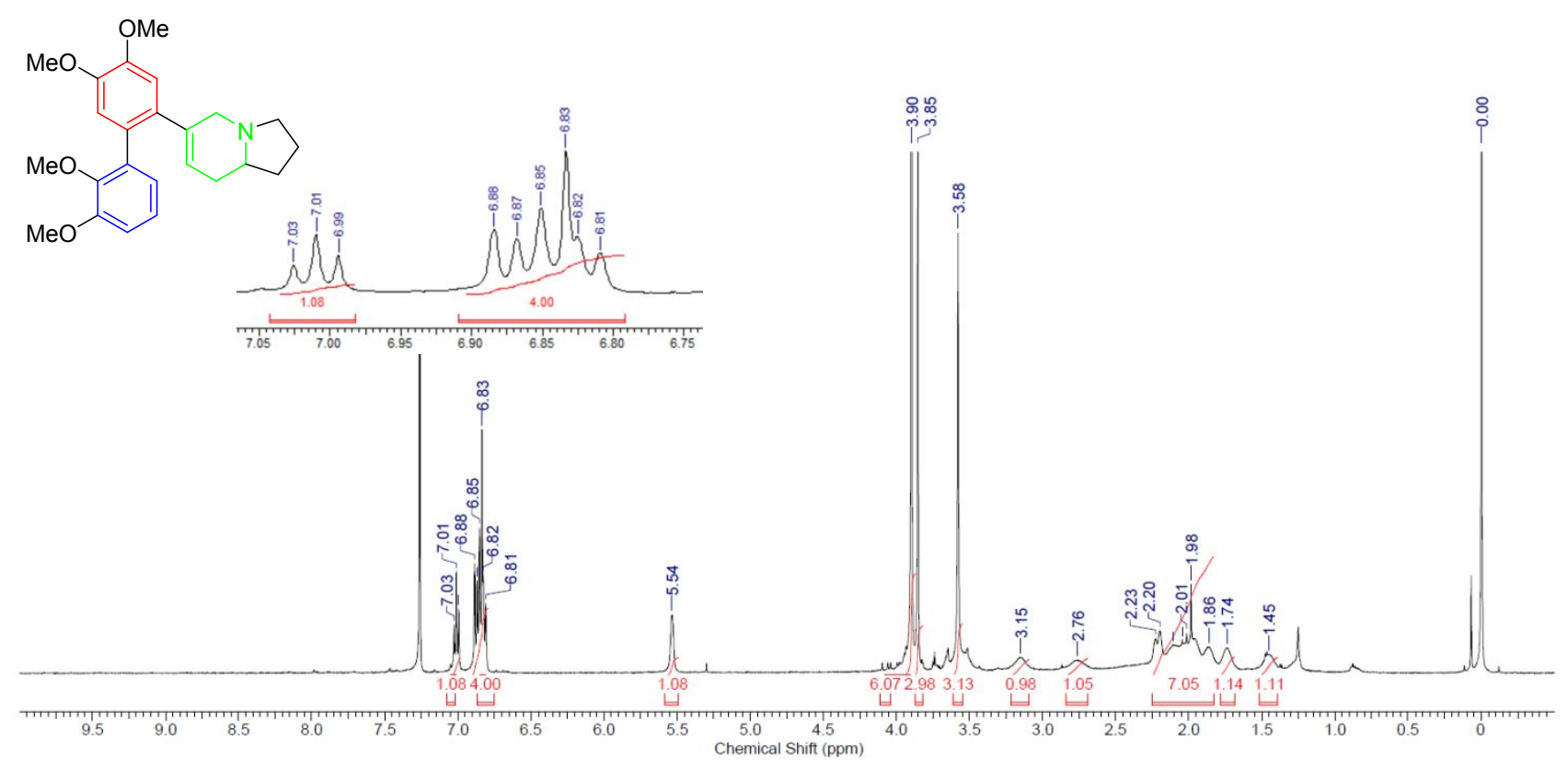

b) ${ }^{13} \mathrm{C}$ NMR Spectrum (in $\mathrm{CDCl}_{3}, 125 \mathrm{MHz}$ )
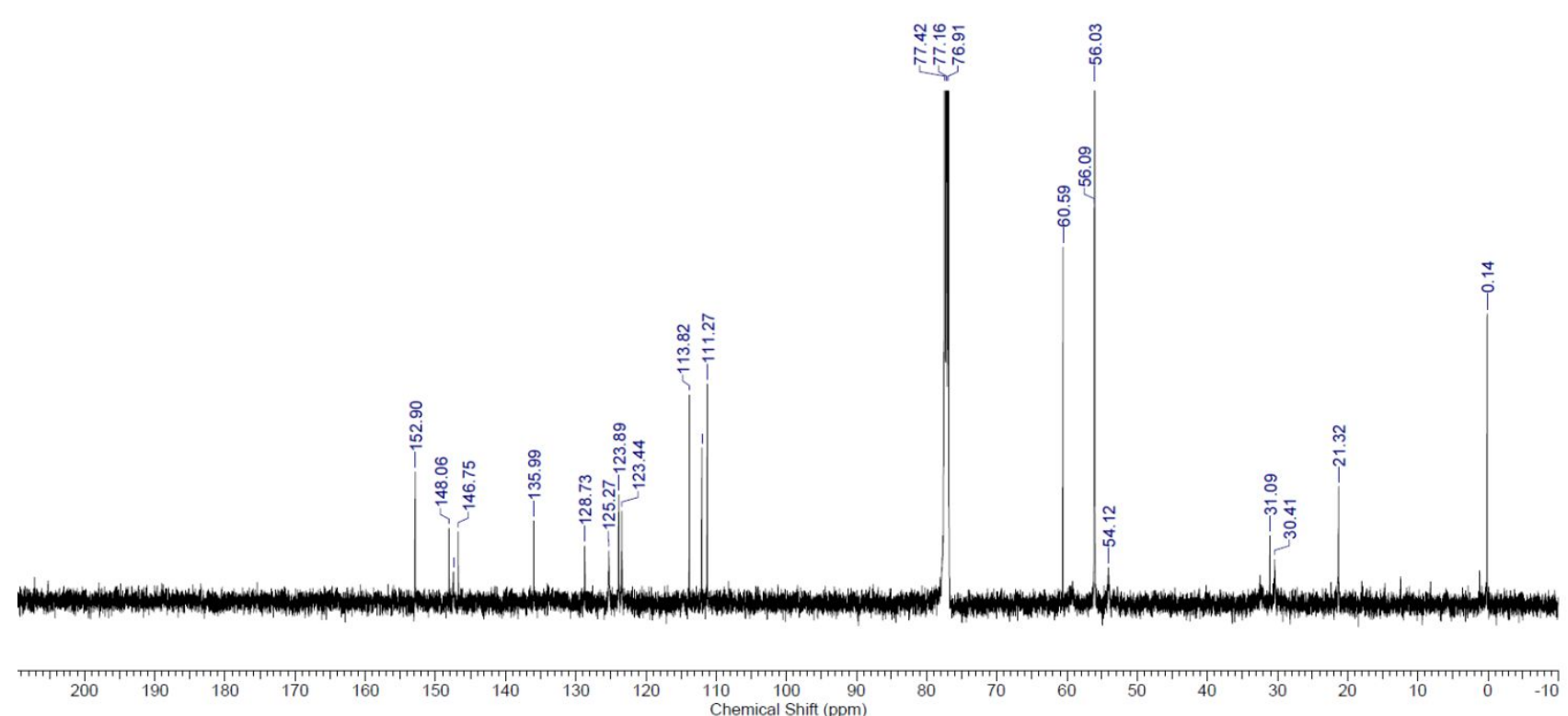
5-18. NMR Spectra of Deoxytylophorine (4)

a) ${ }^{1} \mathrm{H}$ NMR Spectrum (in $\mathrm{CDCl}_{3}, 500 \mathrm{MHz}$ )

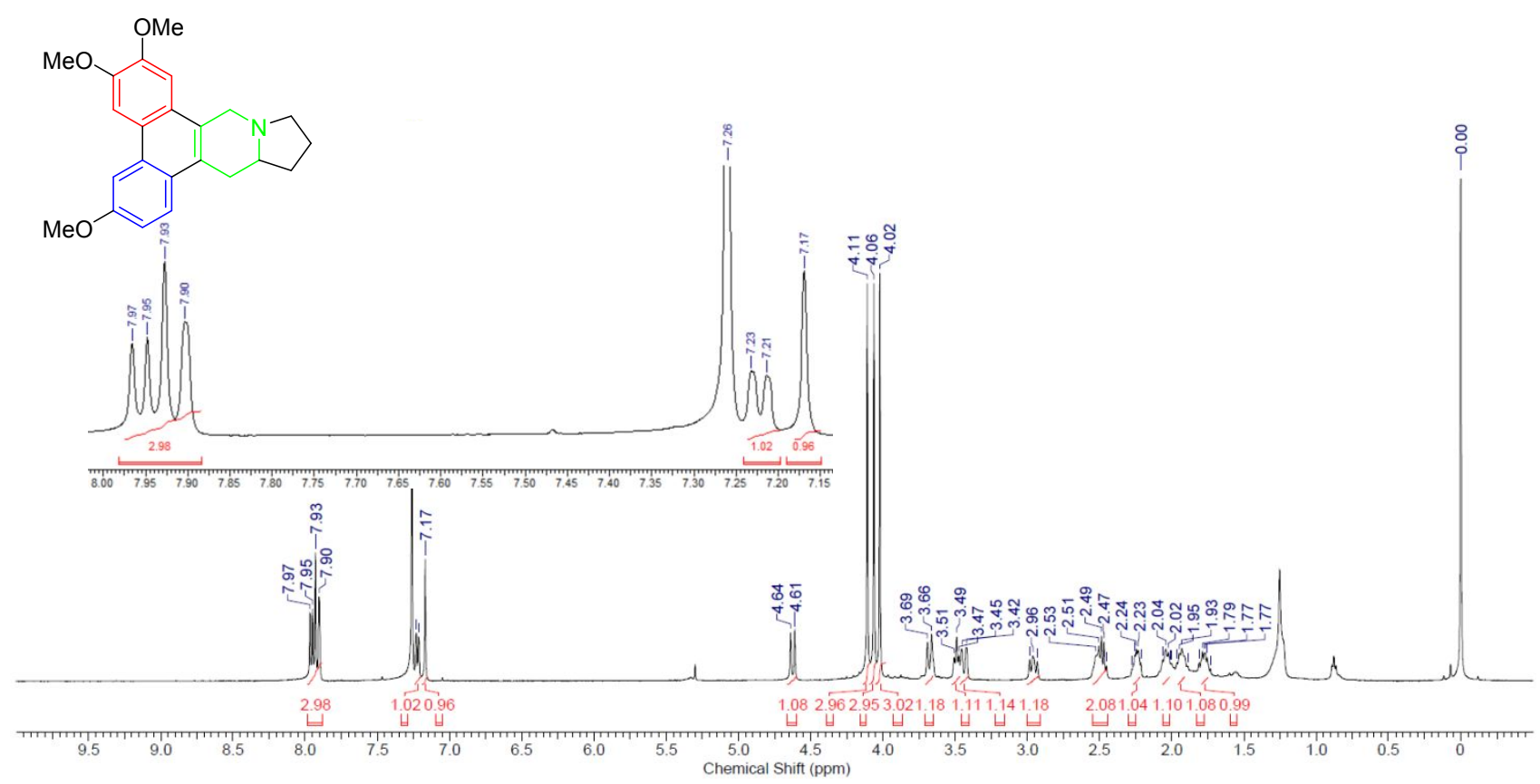

b) ${ }^{13} \mathrm{C}$ NMR Spectrum (in $\mathrm{CDCl}_{3}, 125 \mathrm{MHz}$ )
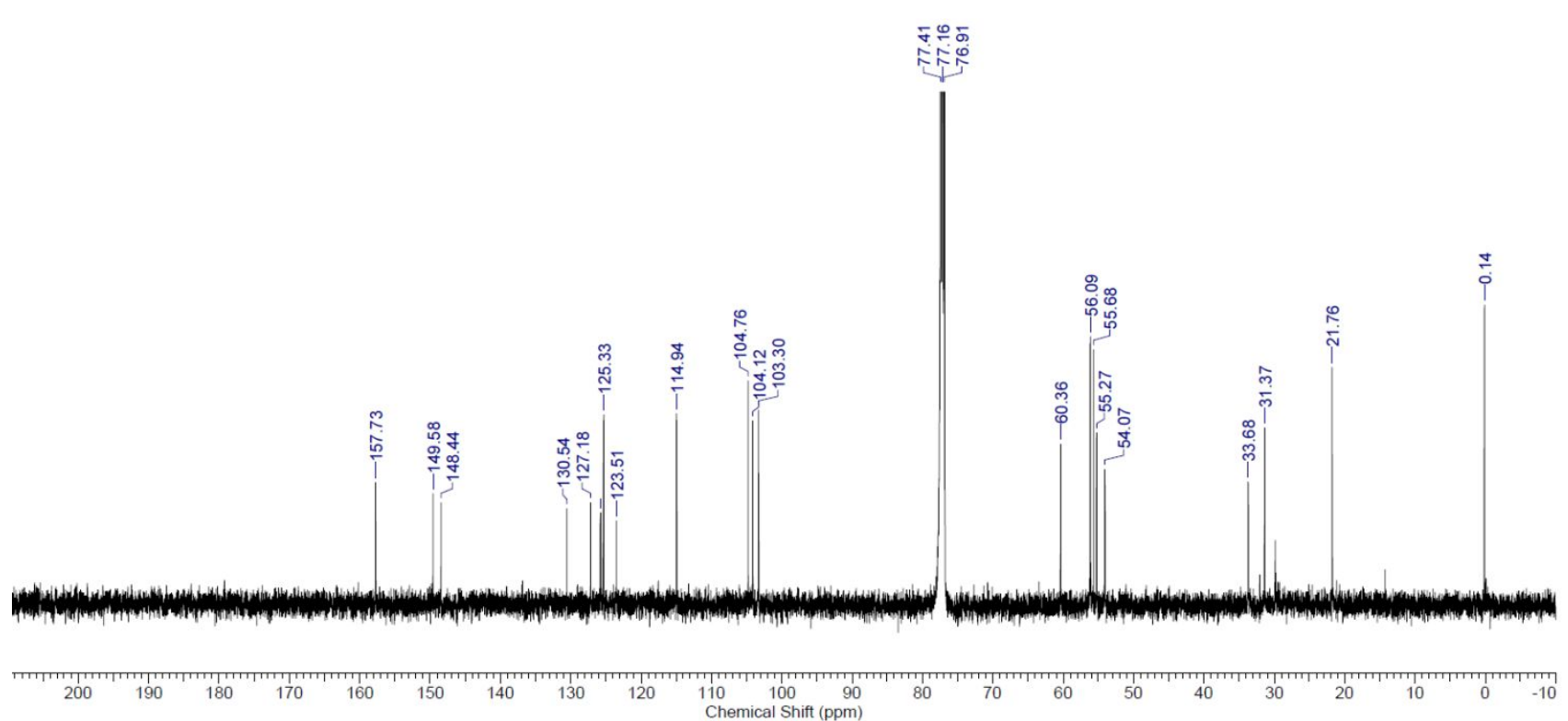
5-19. NMR Spectra of Isotylocrebrine (5)

a) ${ }^{1} \mathrm{H}$ NMR Spectrum (in $\mathrm{CDCl}_{3}, 500 \mathrm{MHz}$ )

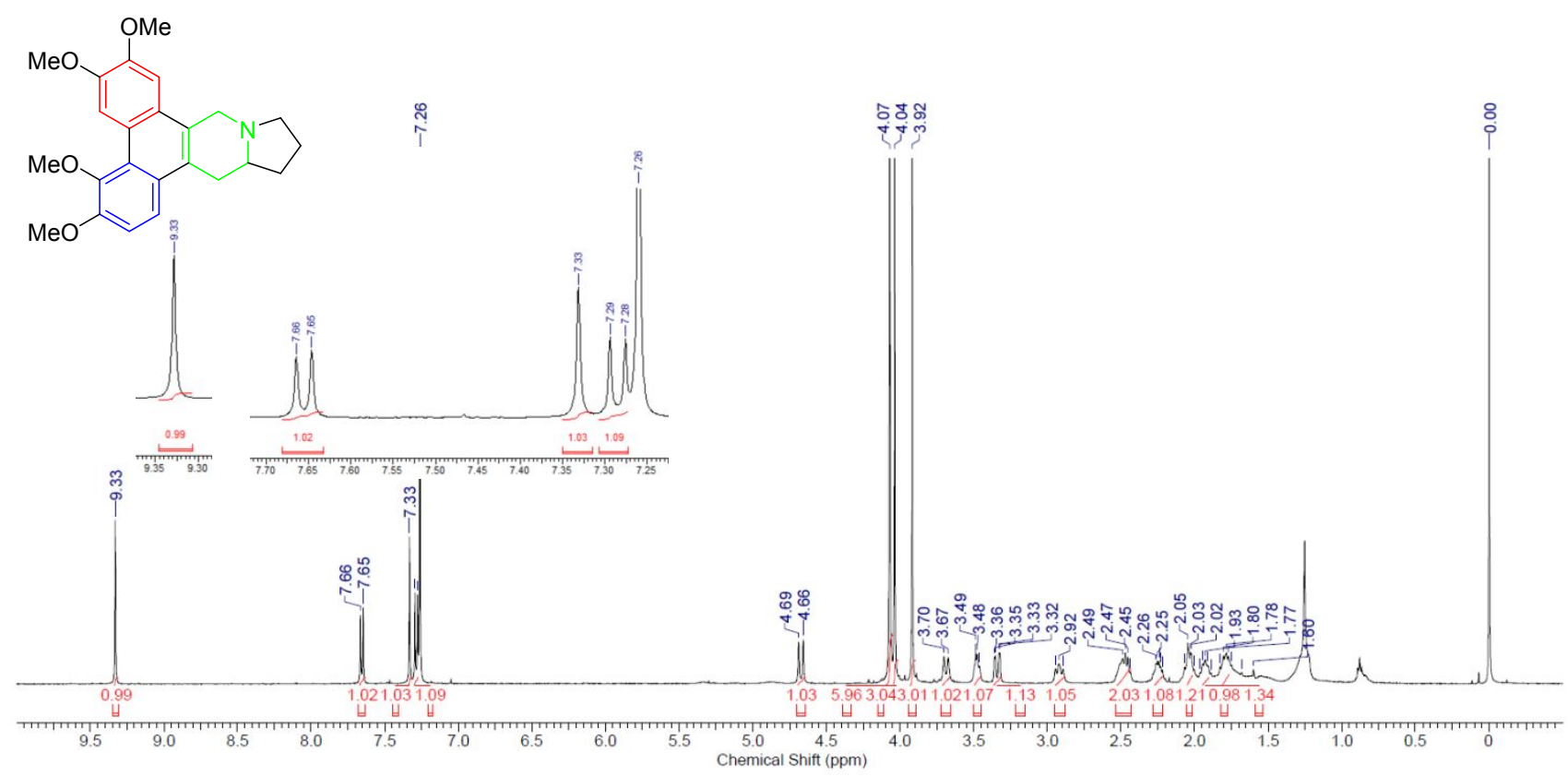

b) ${ }^{13} \mathrm{C}$ NMR Spectrum (in $\mathrm{CDCl}_{3}, 125 \mathrm{MHz}$ )

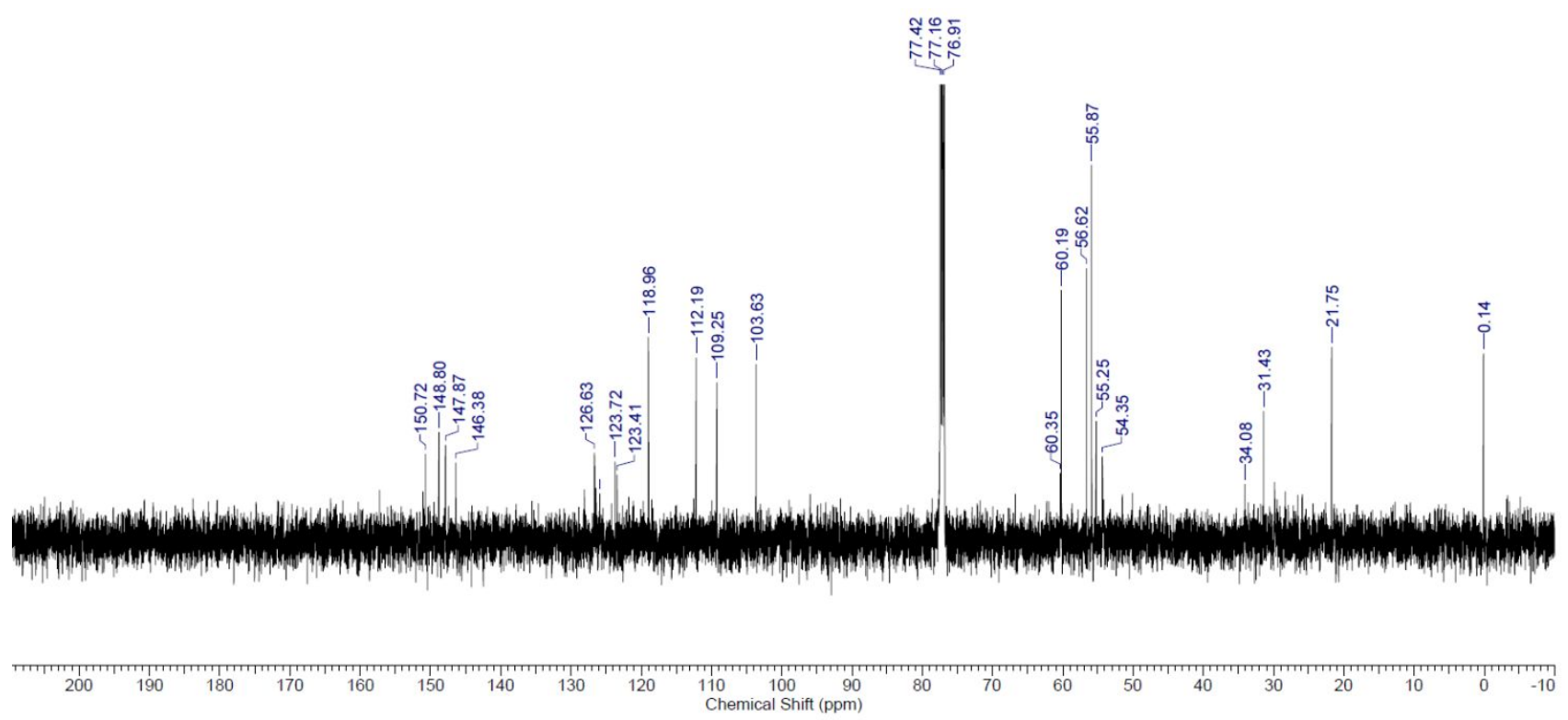

